\title{
Localized phosphorylation of RNA Polymerase II by G1 cyclin-Cdk promotes cell cycle entry
}

Mardo Kõivomägi ${ }^{1}$, Matthew P. Swaffer ${ }^{1}$, Jonathan J. Turner ${ }^{1}$, Georgi Marinov ${ }^{2}$ \& Jan M. Skotheim ${ }^{1}$

${ }^{1}$ Department of Biology, Stanford University, Stanford CA 94305, USA

${ }^{2}$ Department of Genetics, Stanford University, Stanford CA, 94305, USA

\section{Abstract}

The cell cycle is thought to be initiated by cyclin-dependent kinases (Cdk) inactivating 14 transcriptional inhibitors of cell cycle gene-expression(1,2). In budding yeast, the $\mathrm{G} 1$ cyclin CIn3-Cdk1 complex is thought to directly phosphorylate Whi5, thereby releasing the transcription factor SBF and committing cells to division(3-7). Here, we report that Cln3Cdk1 does not phosphorylate Whi5, but instead phosphorylates the RNA Polymerase II

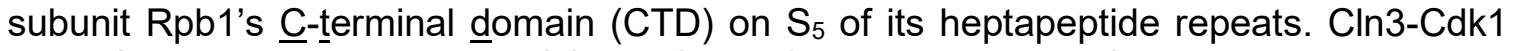
binds SBF-regulated promoters (8) and CIn3's function can be performed by the canonical $\mathrm{S}_{5}$ kinase(9) Ccl1-Kin28 when synthetically recruited to SBF. Thus, Cln3-Cdk1 triggers cell division by phosphorylating Rpb1 at SBF-regulated promoters to activate transcription. Our findings blur the distinction between cell cycle and transcriptional Cdks to highlight

23 the ancient relationship between these processes. 
The eukaryotic cell cycle is driven by a series of cyclin-Cdk complexes that promote cell cycle progression by phosphorylating key substrates(2). The first step of the eukaryotic cell cycle, from $\mathrm{G} 1$ to $S$ phase, has long been thought to require the phosphorylation and inactivation of a transcriptional inhibitor. In human cells, cyclin D-Cdk4,6 phosphorylates the retinoblastoma protein, $\mathrm{Rb}$, and in budding yeast $\mathrm{Cln} 3-\mathrm{Cdk} 1$ is thought to phosphorylate Whi5 $(1,3,4)$. This results in the activation of the E2F and SBF transcription factors, in animal and yeast cells respectively, which then commits cells to division via positive feedback loops (Fig. 1A)(5, 10-15). However, in early to mid G1, cyclin D-Cdk4,6 constitutively hypo-phosphorylates $\mathrm{Rb}(16)$, which is likely insufficient to completely inactivate $\mathrm{Rb}(17)$. As for Whi5, phosphorylation by Cln3-Cdk1 has not previously been observed in vivo. Thus, key mechanistic aspects of the eukaryotic G1/S transition model remain either unknown or untested.

To examine the prevailing model that CIn3-Cdk1 promotes the G1/S transition by progressively phosphorylating and inhibiting Whi5 $(3,4)$, we sought to measure Whi5 phosphorylation in vivo as cells progressed through G1. We used Phos-tag-supplemented SDS-PAGE(18) to separate distinct phospho-isoforms of Whi5 isolated from cells synchronously released from $\mathrm{G} 1$ arrest. This allowed us to resolve not only multiphosphorylated species of Whi5 but also different mono- or di-phosphorylated species, which had never previously been observed (Fig. 1B). Hypophosphorylation of Whi5 is slightly reduced upon release from pheromone arrest, but stays at a constant level until the G1/S transition. At G1/S, Whi5 is rapidly hyper-phosphorylated by $\operatorname{Cln} 1 / 2-\operatorname{Cdk} 1(3,6)$.

Next, we sought to test if Whi5 hypophosphorylation in early G1 is due to Cln3Cdk1. Consistent with Cln3's well-established role in driving G1/S, Whi5 hyperphosphorylation was delayed in $\operatorname{cln} 3 \Delta$ cells synchronously released into the cell cycle as previously reported(19) (Fig. 1C). But, critically, the hypophosphorylation pattern was the same as in WT cells in early- to mid-G1, a time when Cln3 is constitutively expressed and thought to function( 7,20$)$. Furthermore, the hypophosphorylation pattern was unaffected by conditionally expressing CLN3 in G1-arrested cln3 $\Delta b c k 2 \Delta$ cells, or by inhibiting an ATP analog-sensitive Cdk1 (Cdk1 $1^{\text {as }}$ ) in mid-G1 (fig. S1A to C). In contrast, inhibiting $\mathrm{Cdk} 1^{\text {as }}$ in S/G2, after Whi5 was already hyperphosphorylated, caused rapid Whi5 dephosphorylation back to the same G1 hypophosphorylated isoforms (fig. S1D). Taken together, these experiments argue strongly against the prevailing model that Cln3-Cdk1 phosphorylates Whi5 to drive G1/S. Rather, they raise the possibility that CIn3 and Whi5 act as separate inputs regulating SBF activity. Consistent with this separate input model, cln3 $\Delta$ whi5 $\Delta$ cells were larger than whi5 $\Delta$ cells $(3,4)$, and addition of a hyperactive CLN3 allele $(C L N 3 \Delta C)(21,22)$ reduced cell size more than whi5s (Fig. 1D).

If CIn3-Cdk1 functions through SBF but does not target Whi5, it might be present at SBFregulated promoters even in the absence of Whi5. To test this, we performed ChIP-seq analysis of $\mathrm{Cln} 3$ and the SBF components Swi4 and Swi6, all tagged at their endogenous loci with the V5 epitope. CIn3-V5 was found at 85 gene promoters, 84 of which were also bound by SBF (Swi4-V5 and Swi6-V5; Table S3). These sites include key SBF bindingsites in the CLN1 and CLN2 promoters (Fig. 1E, fig. S1E to F), consistent with previous ChIP experiments showing conditionally-expressed CIn3 binding at the CLN2 promoter(8). Furthermore, CIn3-V5 localization to SBF-binding sites, including the CLN2 promoter, did not depend on Stb1 or Whi5, showing that the presence of Cln3 at SBF sites did not depend on its previously assumed target protein (Fig. 1E, fig. S1E to F). 
That $\mathrm{Cln} 3$ binds SBF-regulated promoters suggests that Cln3-Cdk1 phosphorylates a different target involved in SBF-dependent transcription. To explore this possibility, we examined Cln3-Cdk1 kinase activity towards SBF-interacting proteins in vitro. To purify Cln3-Cdk1, we fused CLN3 to CDK1 using a glycine-serine linker $(23,24)(C L N 3-L-C D K 1)$ because $\operatorname{Cln} 3$ is not as tightly bound to Cdk1 as the other yeast cyclins. When the endogenous CLN3 allele was replaced by this fusion allele, cells exhibited no cell cycle defects and were the same size as wild type, suggesting that this fusion complex functions similarly to the wild type allele (fig. S2A). We note that, to the best of our knowledge, this is the first time active Cln3-Cdk1 complexes have been purified to homogeneity. Kinase activity detected in a previously reported purification of Cln3-Cdk1 expressed in insect cells(4), which we repeated, was not due to $\operatorname{Cln} 3$ because this activity was still present in a negative control lacking $\mathrm{Cln} 3$ (see methods; fig. S2B). Consistent with our in vivo phosphorylation data, Cln3-L-Cdk1 poorly phosphorylated Whi5 in vitro, while Cln2-LCdk1 readily hyperphosphorylated Whi5 (Fig. 1F). Moreover, Cln3-L-Cdk1 poorly phosphorylated the SBF-associated proteins(8, 25-27) Swi6, Stb1, and Msa1 in vitro (Fig. $1 \mathrm{G}$ ). This is consistent with a previous study concluding that although the SBF subunit Swi6 was required for $\mathrm{Cln} 3$ function, it was not a direct target of Cln3-Cdk1(28).

The absence of Cln3-dependent phosphorylation on proteins at SBF-regulated promoters, combined with Cln3-Cdk1's lack of in vitro activity against the model Cdk substrate $\mathrm{H} 1$ (Fig. 1G), suggested that Cln3 may promote the G1/S transition independently of Cdk1 kinase activity. To test this possibility, we examined the effect of replacing CLN3 with a $C L N 3$ allele fused to a previously described kinase dead $C D K 1$ allele(29) $\left(C D K 1^{K D}\right.$; Fig. $2 \mathrm{~A}$, fig. S2C). Although $C L N 3-L-C D K 1^{K D}$ rescued the effects of $C \ln 3 \Delta$, immunoprecipitation revealed endogenous Cdk1 bound to the fusion protein (fig. S2D). To prevent this, we introduced a cyclin box mutation to CLN3 that prevents its binding to Cdk1(30) (CLN3 ${ }^{C B M}$; Fig. 2A). Replacing $C L \bar{N} 3$ with $C L N 3^{C B M}$ resulted in a similar large size phenotype as Cln3A, while replacing CLN3 with $C L N 3^{C B M}-L-C D K 1$ resulted in wild type sized cells, indicating that the fusion allows $C \ln 3^{C B M}$ to activate Cdk1 (Fig. 2B). CLN3 ${ }^{C B M}-L-C D K 1^{K D}$ cells lacking $C \ln 3$ activity were similar in size to $\ln 3 \Delta$ cells, suggesting that $C \ln 3-C d k 1$ kinase activity is indeed required to drive the cell cycle through G1/S (Fig. 2B).

To confirm that Cln3-Cdk1 requires kinase activity to promote cell-cycle progression, we examined its function in the context of a simplified cell-cycle control network. We first replaced the CDK1 promoter with the glucose-repressible GAL1 promoter so that all endogenous Cdk1 activity could be conditionally removed. We then proceeded to add back cyclin-Cdk1 fusion proteins expressed from their endogenous cyclin promoters. Cells were viable on glucose when CLB2-L-CDK1 was added to drive M-phase, CLB5-L-CDK1 was added to drive S-phase, and CLN3-L-CDK1 was added to drive G1/S (Fig. $2 \mathrm{C}$ to E). However, addition of CLN3-L-CDK1 ${ }^{K D}$ instead of CLN3-L-CDK1 was insufficient for proliferation, which supports a model in which Cln3-Cdk1 kinase activity promotes the G1/S transition (Fig. $2 \mathrm{C}$ to E). The requirement for Cln3-Cdk1 activity was not alleviated by deletion of WHI5, which is consistent with Whi5 not being a Cln3-Cdk1 substrate (fig. S2F to $G)$.

Having established that Cln3-Cdk1 requires kinase activity to promote the G1/S transition, we sought to identify its substrates. We performed a candidate-based in vitro screen(31), in which we measured the activity of purified Cln3-L-Cdk1 and other yeast cyclin-Cdk1 complexes towards $>20$ Cdk1 target proteins (Fig. 3A to C; fig. S3A to D). By far the most specific target for Cln3-L-Cdk1 was the RNA polymerase II subunit Rbp1, which contains a C-terminal unstructured region (CTD) with multiple heptapeptide repeats (26 in yeast, 
12552 in humans) of the sequence $\mathrm{Y}_{1} \mathrm{~S}_{2} \mathrm{P}_{3} \mathrm{~T}_{4} \mathrm{~S}_{5} \mathrm{P}_{6} \mathrm{~S}_{7}$ (32) (Fig. 3C). Truncations of Rpb1 to first

126

127

128

129

130

131

132

133

134

135

136

137

138

139

140

141

142

143

144

145

146

147

148

149

150

151

152

153

154

155

156

157

158

159

160

161

162

163

164

165

166

167

168

169

170

171

172

173

174

175 isolate the unstructured $\mathrm{C}$-terminal region and then to remove the regions on either side of the CTD heptad repeats did not reduce phosphorylation, implying that Cln3-Cdk1 directly targets one or multiple residues inside the heptapeptide repeats independently of the adjacent unstructured regions (Fig. 3D to E).

Phosphorylation of the different residues within these heptad repeats by the canonical transcriptional kinases regulates transcriptional initiation, elongation, and termination(32). To compare Cln3-Cdk1 with the four known transcriptional kinases, we applied our in vitro approach to purify Ccl1-L-Kin28, Bur2-L-Bur1, Ctk2-L-Ctk1 and Ssn8-L-Ssn3, which correspond to human Cdk7, Cdk9, Cdk12, and Cdk8 complexes respectively(33). This revealed Cln3-Cdk1, Kin28, Ssn3 and Ctk1 all phosphorylate residues inside the CTD repeats independently of the adjacent unstructured regions (fig. S3E to G). In contrast, Bur1 did not phosphorylate the CTD repeats without the C-terminal non-repeat element (Fig. 3E), consistent with published work(34).

To determine the function of Cln3-Cdk1 phosphorylation of the CTD, we sought to identify the specific target residues. To do this, we generated a series of model substrates comprising a GST sequence followed by four wild type or mutant CTD consensus repeats (GST-4CTD) (Fig. 3F). Of the possible Cdk phosphorylation site mutants, only mutation of the serine 5 residue prevented phosphorylation (GST-4CTD $5 \mathrm{~A}$ ). Conversely, the addition of $S_{5}$ to a repeat region lacking all serines restored phosphorylation (GST-4CTD S5 $_{5}$ in Fig. $3 G$ ). Similar results were found when this set of substrates was phosphorylated by Ccl1L-Kin28, but not when phosphorylated by other cyclin-Cdk1 complexes (Fig. 3G to H). That CIn3-Cdk1 could function as an $\mathrm{S}_{5}$ CTD kinase is consistent with the reported genetic interactions between the canonical $S_{5}$ CTD kinase Kin28 and Cdk1(35). To determine if other residues inside the CTD heptad might be responsible for Cln3-Cdk1 specificity, we performed in vitro kinase assays with additional GST-4CTD substrates in which $Y_{1}, P_{3}, T_{4}$, or $\mathrm{P}_{6}$ were substituted with alanines. Phosphorylation was decreased by $Y_{1}, P_{3}$, and $P_{6}$ mutations, but not by $\mathrm{T}_{4}$ mutation (Fig. 3G; fig. S3E). The effect of the $P_{6}$ alanine substitution was expected because Cdk1 is a proline directed kinase, while the $\mathrm{P}_{3}$ requirement 2 residues $\mathrm{N}$-terminal to the phosphorylation site is similar to that found to enhance phosphorylation by CIn2-Cdk1 complexes(31). However, the $\mathrm{Y}_{1}$ requirement was surprising and suggests a highly specific substrate preference for the active site of CIn3Cdk1. Taken together, our results show that Cln3-Cdk1 likely phosphorylates $S_{5}$ and that this phosphorylation depends on the local amino acid sequence (Fig. 3I).

That CIn3 localizes to specific SBF-regulated promoters, and that Cln3-L-Cdk1 functions as an $\mathrm{S}_{5}$ CTD kinase in vitro, suggests a model in which Cln3-Cdk1 promotes transcription of SBF-regulated genes by phosphorylating the CTD of Rpb1 at their promoters. In this model, Cln3-Cdk1 should be responsible for only a subset of the global $S_{5}$ CTD phosphorylation. To test this model, we first replaced endogenous KIN28 with the KIN28 ${ }^{\text {is }}$ allele, which expresses a version of Kin28 that can be irreversibly inhibited by a covalentlybinding small molecule(36). Addition of the Kin28 inhibitor reduced $S_{5}$ CTD phosphorylation by $60 \pm 3 \%$, and deletion of CLN3 further reduced phosphorylation by another $15 \pm 5 \%$ in cells in the G1 phase of the cell cycle, showing that Cln3-Cdk1 phosphorylates $\mathrm{S}_{5}$ in the CTD repeats in vivo (Fig. $3 \mathrm{~J}$ to L, fig. S4; see methods).

Taken together, our work supports a model in which Cln3-Cdk1 promotes the G1/S transition by phosphorylating $S_{5}$ in the RNA polymerase II CTD at SBF-regulated promoters (Fig. 4A). This model predicts that we should be able to bypass the requirement 
176 for Cln3 by providing an alternative source of $S_{5}$ phosphorylation to SBF-regulated promoters. To test this, we used a rapamycin-dependent inducible binding system to conditionally recruit a fusion protein of the canonical CTD $S_{5}$ kinase Ccl1-L-Kin28 to SBF via its Swi6 or its Swi4 subunit (Fig. 4B, fig. S5A and B). We note that all these strains contain the tor1-1 fpr1s mutations so that growth is not affected by rapamycin(37). Strikingly, recruitment of Ccl1-L-Kin28 to SBF fully rescues the size and cell cycle phenotypes of $c / n 3 \Delta$ cells and this rescue is dependent on Kin28 kinase activity (Fig. 4C to $\mathrm{E}$, fig. S5C to F). This rescue was not due to Kin28 phosphorylation of Whi5 (fig. S5G to J).

Thus, CIn3-Cdk1 promotes the first step in the budding yeast cell cycle by directly phosphorylating RNA Polymerase II at SBF-regulated genes. Surprisingly, Cln3-Cdk1 did not phosphorylate its expected target, the SBF inhibitor Whi5, or any other proteins on the SBF transcription factor complex. Moreover, our screen suggests that Cln3-Cdk1 has few, if any, targets other than the RNA Polymerase II subunit Rpb1's CTD. Cln3-Cdk1's extreme specificity - for a single target driving the first step of the cell cycle-may help order cell cycle events(31). If, as we suspect, Cln3 has no targets driving replication, spindle pole body duplication, cell polarization, or any other cell cycle event, the level of CIn3 can be used to exclusively modulate the G1/S transition without risking premature triggering of downstream cell cycle events.

That Cln3 and Whi5 serve as separate inputs to SBF activity can be rationalized by their potentially separate functions. In G1, Whi5 concentration directly reflects cell size because Whi5 is a stable protein and a constant number of Whi5 molecules is synthesized in $\mathrm{S} / \mathrm{G} 2 / \mathrm{M}$ phases independent of cell size and growth conditions $(20,38)$. As cells grow in $\mathrm{G} 1$, Whi5 is then diluted so that its concentration is determined by cell size. In contrast, Cln3 concentration is constant in $\mathrm{G} 1$ as cells grow in a given condition, but this constant G1 concentration is higher when cells are growing rapidly, as in glucose, and lower, when cells are growing more slowly, as in ethanol $(7,39-43)$. Thus, Cln3 and Whi5 may independently reflect cell growth and size, respectively.

While the cell cycle Cdks and the transcriptional Cdks are all part of the same kinase family, their functions are largely thought to have diverged along their separate branches $(44,45)$. Our work here breaks down the previous dichotomy of cell cycle and transcriptional Cdks and shows how cell cycle Cdks can directly activate transcription at specific target genes to drive a cell cycle transition. The functional overlap of cell cycle and transcriptional Cdks has already been pointed to by the dual function of the Kin28 orthologs, Msc6 and Cdk7 in fission yeast and vertebrate cells, respectively. They both activate the cell cycle Cdks by phosphorylating their T-loops while also activating transcription as a global $S_{5}$ CTD kinase(46-48). In addition, Cdk1 and Cdk2 were identified as the first RNA Pol II CTD kinases in vitro, but if they function in such a manner in vivo remains unknown(49). That the two branches of Cdks that regulate cell division and transcription have overlapping functions suggests the possibility that their primordial ancestor regulated both processes. These functions would then have been partially lost along the two divergent branches. Thus, our discovery that yeast Cln3-Cdk1 drives cell cycle progression by directly activating transcription may reflect an ancient link between basic biosynthetic processes like transcription and the control of cell division. 


\section{FIGURES \& CAPTIONS}

Figure 1
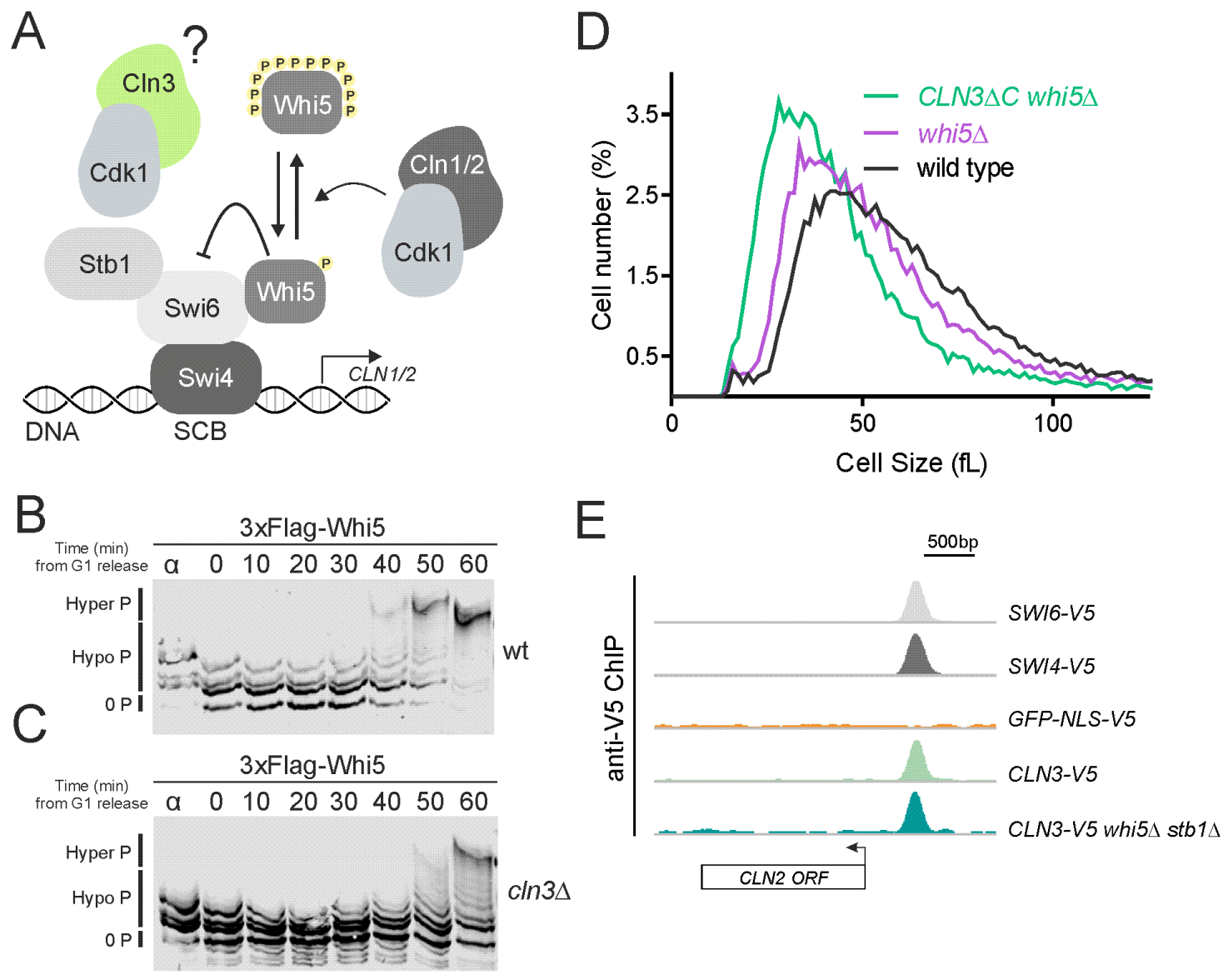

$\mathrm{F}$

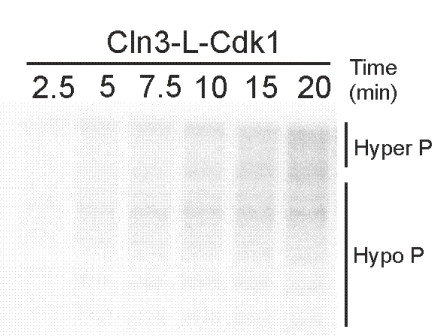

Whi5

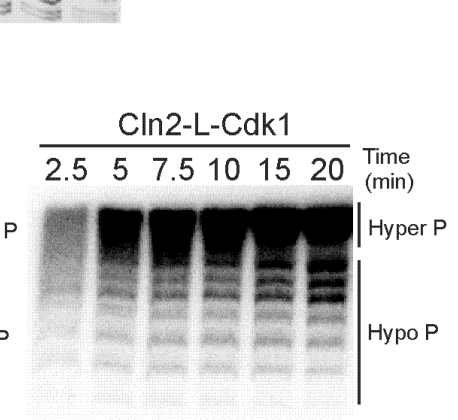

Whi5

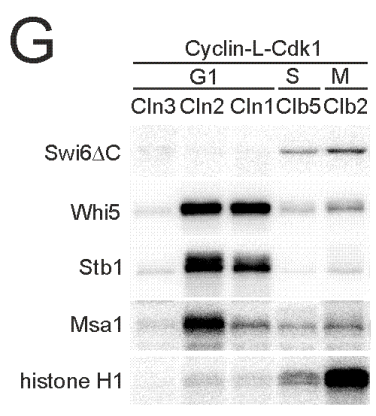

Fig. 1. Cln3-Cdk1 binds SBF, but does not phosphorylate the transcriptional inhibitor Whi5. (A) Schematic of the budding yeast G1/S regulatory network. (B) Phostag immunoblot time course measuring distinct hypo- and hyper-phosphorylated isoforms of 3xFlag-Whi5 after release from a G1 pheromone arrest. (C) Phos-tag immunoblot time course as in (B) for $\operatorname{cln} 3 \Delta$ cells. (D) Cell size distributions measured by Coulter counter for the indicated genotypes. Cells were grown on synthetic complete media with $2 \%$ glucose. (E) anti-V5 ChIP-seq signal of the indicated genotypes at the CLN2 locus. CLN2 is an SBF target, whose expression drives the G1/S transition. See fig. S1E to $F$ and methods for more details. (F) Autoradiographs of in vitro Whi5 phosphorylation time courses by Cln2L-Cdk1 and Cln3-L-Cdk1 fusion proteins, where L denotes a glycine serine linker (see 
237 methods for purification and verification of Cln3-L-Cdk1 activity; fig. S3C). Whi5 phospho238 isoforms were resolved using Phos-tag SDS-PAGE. All reactions contain equal amounts 239 of the indicated cyclin-L-Cdk1 complexes. (G) Autoradiographs of in vitro phosphorylation 240 of SBF-interacting proteins and histone $\mathrm{H} 1$ by the indicated cyclin-L-Cdk1 complexes. All 241 reactions contain equal amounts of the respective cyclin-L-Cdk1 complexes. 


\section{Figure 2}

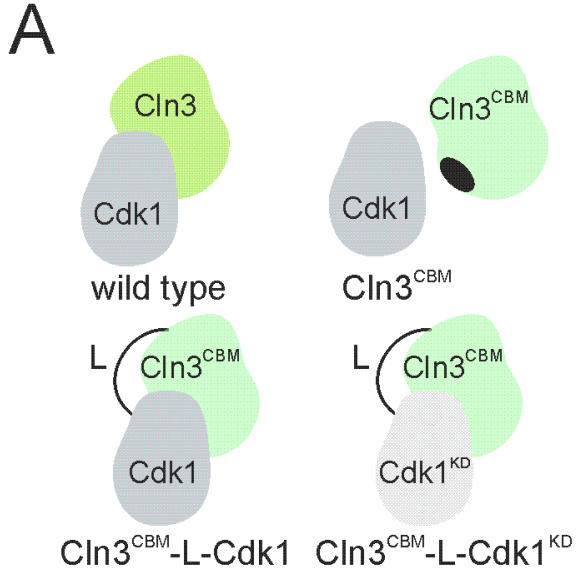

C

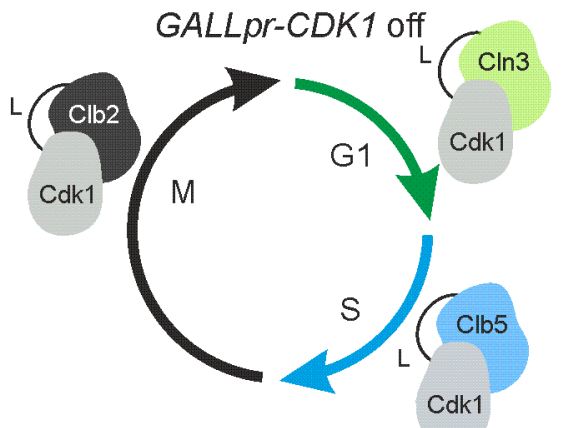

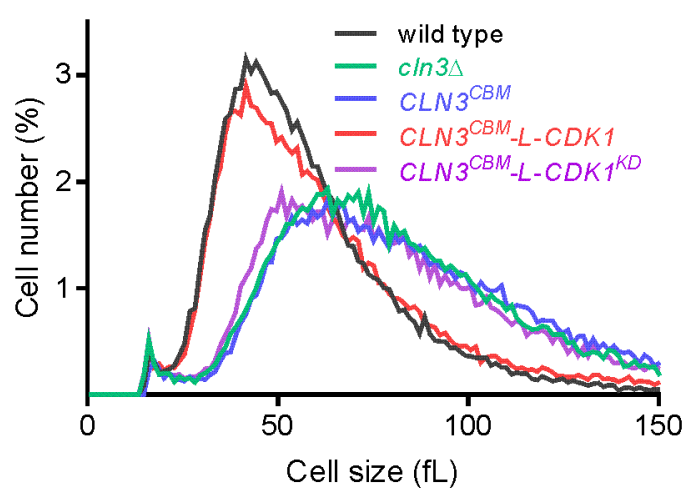

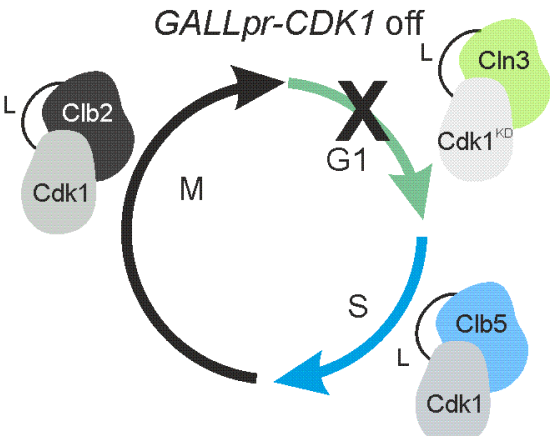

$\mathrm{D}$

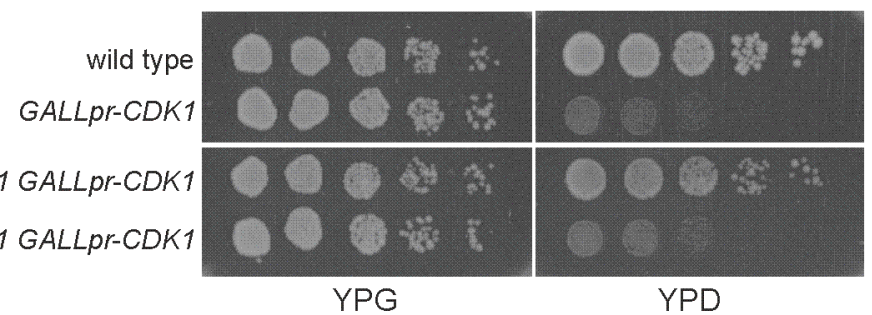

$\mathrm{E}$

CLN3/CLB5/CLB2-L-CDK1 GALLpr-CDK1 CLN3-L-CDK1 ${ }^{K D}$ CLB5/CLB2-L-CDK1 GALLPr-CDK1

YPG

YPD

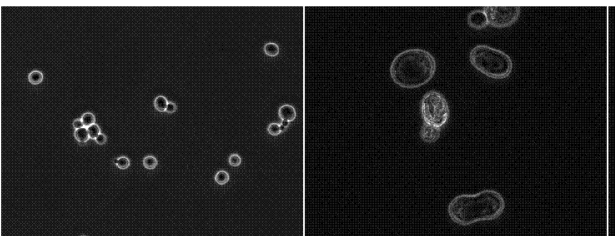

wild type

GALLPr-CDK1 off

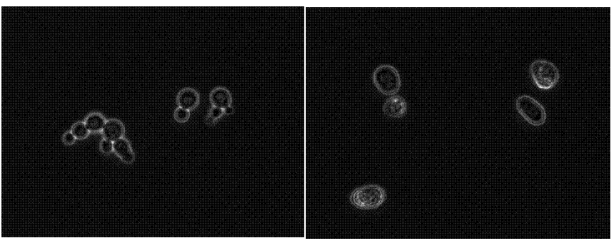

GALLPr-CDK1 off

GALLpr-CDK1 off CLN3pr-CLN3-L-CDK1 CLN3pr-CLN3-L-CDK1 ${ }^{K D}$ CLB5pr-CLB5-L-CDK1 CLB5pr-CLB5-L-CDK1

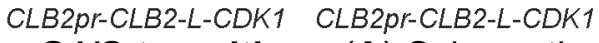

Fig. 2. CIn3-Cdk1 kinase activity promotes the G1/S transition. (A) Schematic of the different Cln3-Cdk1 complexes used. Cln3 ${ }^{\mathrm{CBM}}$ denotes a cyclin box mutant that does not bind Cdk1 unless fused via the linker, L. Cdk $1^{\mathrm{KD}}$ denotes a kinase dead mutant of Cdk1. (B) Cell size distributions measured by Coulter counter for the indicated genotypes. Cells were grown on synthetic complete media $+2 \%$ glucose. (C) Schematic of the experimental 
249 design shown in (D). The endogenous CDK1 promoter was replaced with the galactose250 inducible GALL promoter (GALLpr). CLN3, CLB5, and CLB2 were then fused at their 251 endogenous locus to CDK1. (D) Spot viability assays of WT and GALLpr-CDK1 strains 252 the on YPG (GALLpr ON) or YPD (GALLpr OFF). The triple cyclin-CDK1 fusion rescues 253 CDK1 repression only if CLN3 is fused to an active CDK1. (E) Phase contrast images of 254 cells of the indicated genotypes after GALLpr-CDK1 repression. 


\section{Figure 3}

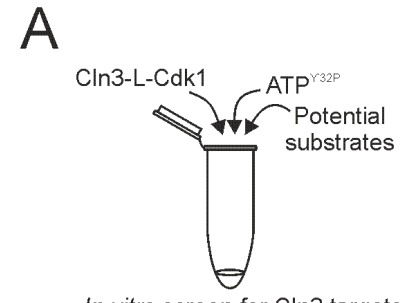

In vitro screen for Cln3 targets
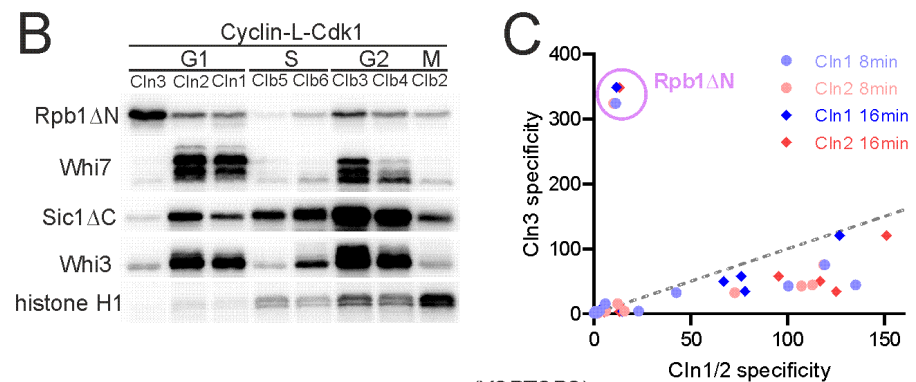

$\mathrm{D}$
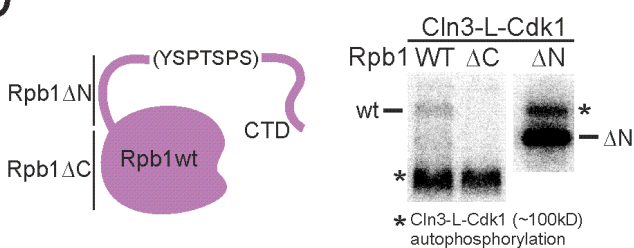

$F_{\text {GST-4CTD: }}$
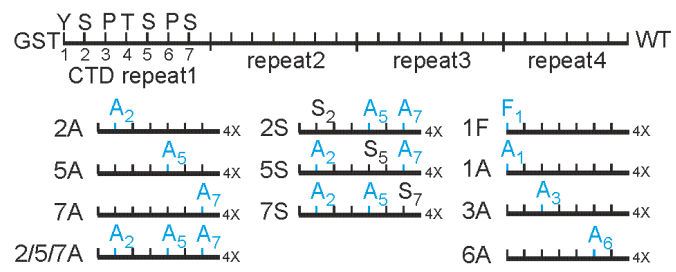

$G$

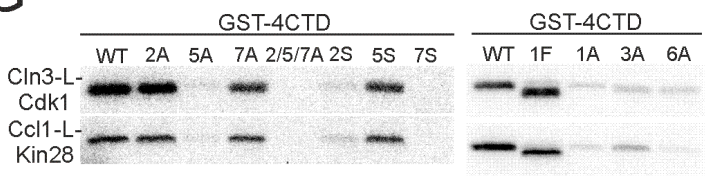

E

(YSPTSPS)

$\mathrm{Cln} 1 / 2$ specificity
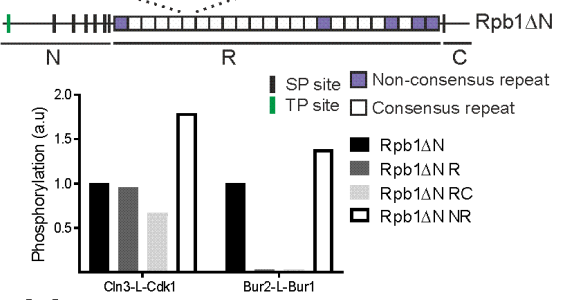

H

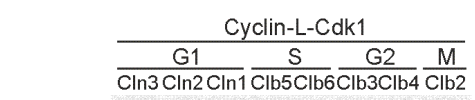

GST-4CTD - - -

GST-4CTD-5A

GST-4CTD-5S =

GST-H1

histone $\mathrm{H} 1$
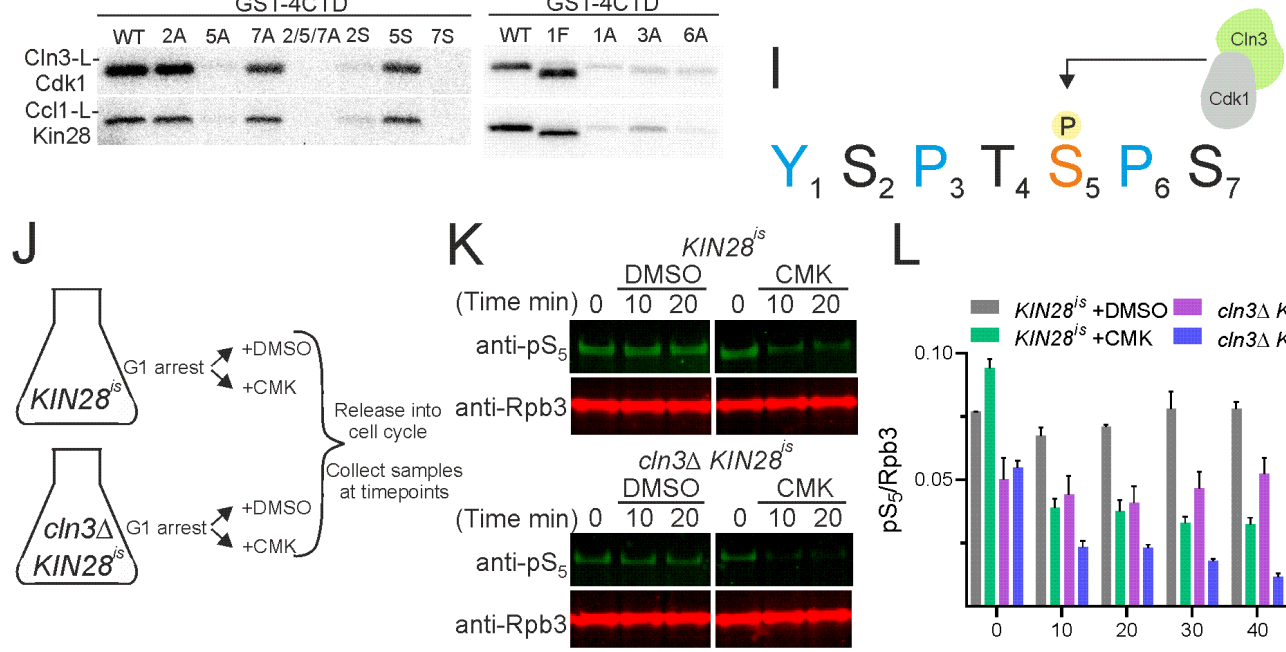

L

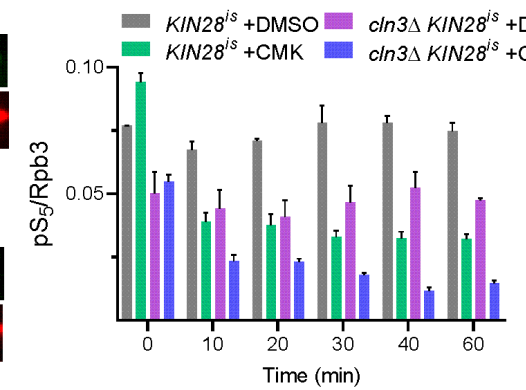

Fig. 3. Cln3-Cdk1 phosphorylates the serine 5 residue in the Pol II subunit Rpb1's C-terminal domain repeats. (A) Schematic of in vitro candidate based screen for CIn3Cdk1 targets. (B) Autoradiographs of in vitro phosphorylation of a subset of candidate substrates by the indicated cyclin-L-Cdk1 complexes. All reactions contain equal amounts of the indicated cyclin-L-Cdk1 complexes. (C) Quantification of in vitro phosphorylation specificity by CIn3-L-Cdk1 compared to Cln1-L-Cdk1 or CIn2-L-Cdk1. Specificity is defined as the ratio of activity towards the indicated substrate relative to the activity towards the Cdk1 model substrate histone $\mathrm{H} 1$. Each point corresponds to a single substrate $(n=20)$ 
265 and quantifications from two time points are plotted. Dotted line denotes $\mathrm{x}=\mathrm{y}$ where CIn3266 L-Cdk1 substrate specificity is equally to Cln1-L-Cdk1 or Cln2-L-Cdk1 specificity. See methods and Table S4 for measurements. (D) Autoradiographs of in vitro phosphorylation by CIn3-L-Cdk1 of Rpb1 and Rpb1 truncations (WT denotes full length; $\Delta \mathrm{C}$ denotes the $1453 \mathrm{~N}$-terminal residues; $\Delta \mathrm{N}$ denotes the $280 \mathrm{C}$-terminal residues). We note the low yield of full length Rpb1 $(\sim 192 \mathrm{kD})$ results in a lower phosphorylation signal than the $\Delta \mathrm{N}$. Full length and $\triangle \mathrm{C}$ Rpb1 were resolved on $6 \%$ SDS PAGE gels, while $\Delta \mathrm{N}$ was resolved on a separate $10 \%$ SDS PAGE gel. (E) Autoradiograph quantification from in vitro kinase assays phosphorylating $\mathrm{Rpb} 1 \Delta \mathrm{N}$ and $\mathrm{Rpb} 1 \Delta \mathrm{N}$ truncations by $\mathrm{Cln} 3-\mathrm{L}-\mathrm{Cdk} 1$ and the transcriptional kinase Bur2-L-Bur1. See fig. S3G for autoradiograph images including data for other transcriptional kinases. (F) Schematic of GST epitope model substrates used in (G), containing 4 copies of the CTD repeat. (G) Autoradiographs of in vitro phosphorylation of CTD repeats with the indicated amino acid substitutions by CIn3-L-Cdk1 and Ccl1-LKin28. (I) Schematic showing $S_{5}$ specific phosphorylation by Cln3-Cdk1. (J) Schematic of experimental design for (K-L). Kin28is or Kin28is cln3s cells were released from G1 pheromone arrest into DMSO or $\mathrm{CMK}$. Kin28 ${ }^{\text {is }}$ contains an active site mutant rendering it sensitive to covalent inhibition by the small molecule CMK. (K) Representative immunoblots for total cellular phosphorylated Rpb1-CTD $S_{5}$ (H14 antibody) and total cellular RNA polymerase II (Rpb3) after release from G1 pheromone arrest into DMSO or CMK. See fig. S4C for full immunoblots. (L) Quantification of total cellular phosphorylated Rpb1-CTD $S_{5}$ (H14 antibody) normalized to total cellular RNA polymerase II (Rpb3). Mean \pm S.E.M. is plotted, calculated from two independent biological replicates. 


\section{Figure 4}

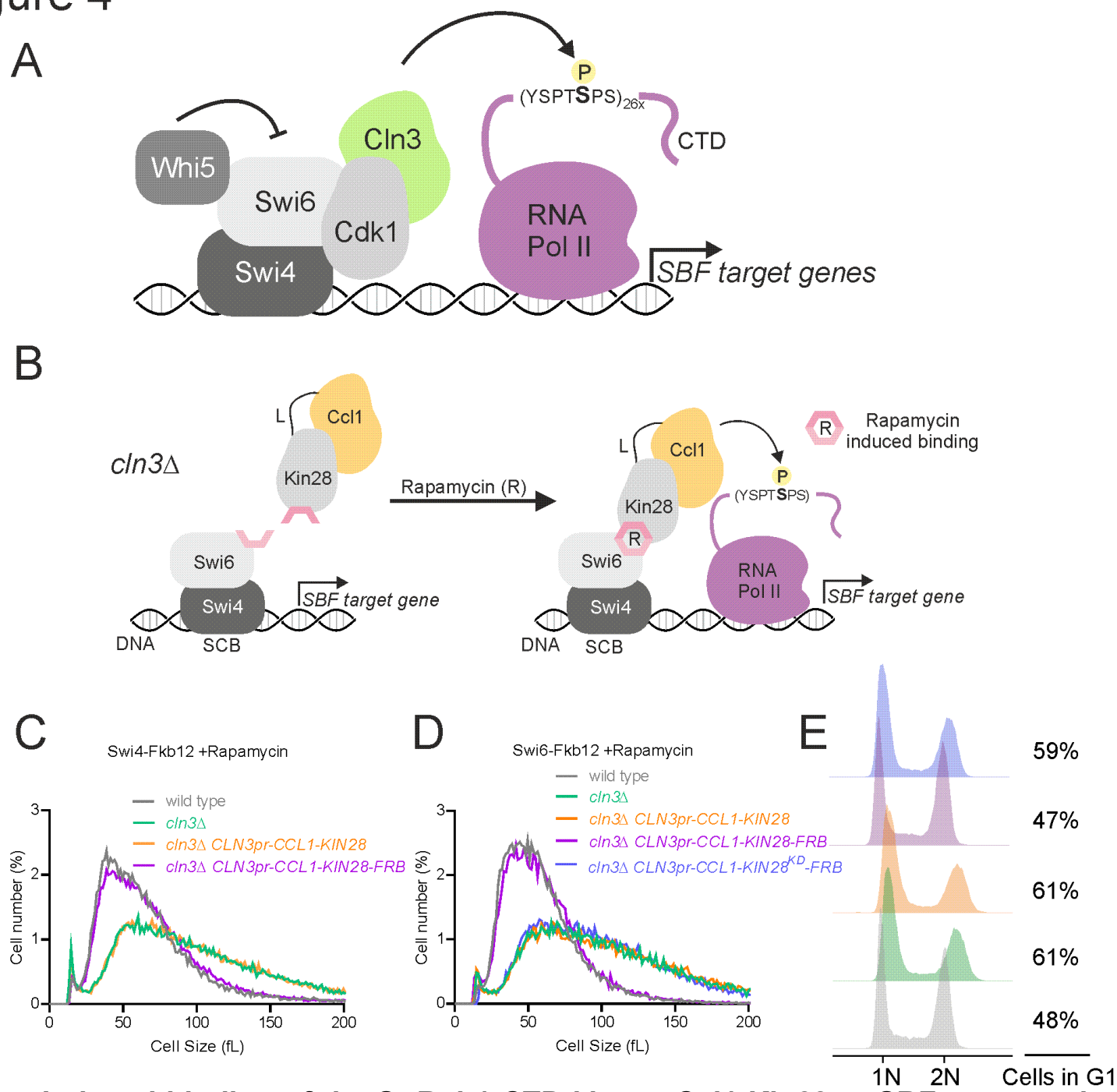

Fig. 4. Induced binding of the $S_{5}$ Rpb1 CTD kinase Ccl1-Kin28 to SBF rescues the cell cycle defects of $\operatorname{cln} 3 \Delta$ cells. (A) Model in which Cln3-Cdk1 activates transcription at SBF-dependent promoters by phosphorylating Rpb1's CTD on $S_{5}$. (B) Schematic of experiment in (c-e): conditional recruitment of Ccl1-L-Kin28 to SBF using the rapamycin inducible binding system. (C-D) Cell size distributions measured by Coulter counter for the indicated genotype. Ccl1-L-Kin28 fusion proteins were expressed from a genomically integrated copy of the CLN3 promoter. Ccl1-L-Kin28-FRB was recruited to SBF via Swi4 (C) or Swi6 (D) upon rapamycin treatment. Ccl1-L-Kin28 lacking FRB is not recruited. All strains were grown on synthetic complete media with $2 \%$ glucose and with $1 \mu \mathrm{g} / \mathrm{ml}$ rapamycin or DMSO. KIN28 ${ }^{K D}$ denotes a kinase-dead $K I N 28$, whose recruitment to SBF content of the cells in (D). 
300

301

302

303

304

305

306

307

308

309

310

311

312

313

314

315

316

317

318

319

320

321

322

323

324

325

326

327

328

329

330

331

332

333

334

335

336

337

338

339

340

341

342

343

344

345

346

347

348

349

350

\section{TABLES AND TABLE CAPTIONS}

Table S1: Saccharomyces cerevisiae strains used in this study. Parentheses denote plasmids, unless otherwise noted all other genotypes represent genomic integration. Strains and plasmids were made using standard methods. DOM0090 was obtained from David Morgan, JE103 was obtained from Jennifer Ewald(50), and K14708 was obtained from Euroscarf(37). $C L N 3^{C B M}$ denotes the mutations to the cyclin box of $C L N 3(30)$. $K I N 28^{\text {nointron }}$ denotes an allele of KIN28 where the intron was removed. CLN3- $\triangle C$ denotes an allele of CLN3 lacking the C-terminal unstructured region, i.e., the allele is truncated at bp1197. WHI5 ${ }^{C D K}$ denotes an allele of WHI5 where two non-Cdk1 sites that we found to be phosphorylated in $\mathrm{G} 1$ were removed (Kõivomägi et al in preparation). $C D K 1^{K D}$ denotes a kinase dead allele of $C D K 1$ where $K 40$ was mutated to $L(29)$. KIN28 is denotes a $K I N 28$ allele that can be covalently inhibited(36). SIC1dC denotes a SIC1 allele truncated at bp645(51). RPB1dN (bp4360-5202) and RPB1dC (bp1-4359) denote truncations of either the $\mathrm{N}$ - or $\mathrm{C}$-terminal unstructured regions. Subscript numbers denote the basepairs present of the gene indicated that were present.

Strain Description

DOM0090 (W303) MATa bar1::HisG

MKy9

MKy12

MKy350

MKy38

MKy226

MKy770

MKy759

MKy760

MKy761

MKy762

MKy763

MKy669

MKy752

MKy753

MKy754

MKy31-1

MKy92-1

MKy347

MKy348

MKy21

MKy76

MKy77

MKy554

MKy555

MKy653

MKy645

MKy637

MKy627

MKy812

JE103

(W303) MATa ksp1::TRP1

MKy9 [pGAL1-3Flag-CLN3-L-CDK1-pRS425]

MKy9 [pGAL1-3Flag-CLN3 ${ }^{C B M}$-L-CDK1-pRS425]

MKy9 [pGAL1-3Flag-CLN2-L-CDK1-pRS425]

MKy9 [pGAL1-3Flag-CLN1-L-CDK1-pRS425]

MKY9 sic1::URA3

MKy770 [pGAL1-3Flag-CLB5-L-CDK1-pRS425]

MKy770 [pGAL1-3Flag-CLB6-L-CDK1-pRS425]

MKy770 [pGAL1-3Flag-CLB3-L-CDK1-pRS425]

MKy770 [pGAL1-3Flag-CLB4-L-CDK1-pRS425]

MKy770 [pGAL1-3Flag-CLB2-L-CDK1-pRS425]

MKy9 [pGAL1-3Flag-CCL1-L-KIN28 ${ }^{\text {nointron }}$-pRS425]

MKy9 [pGAL1-3Flag-BUR2-L-BUR1-pRS425]

MKy9 [pGAL1-3Flag-CTK2-L-CTK1-pRS425]

MKy9 [pGAL1-3Flag-SSN8-L-SSN3-pRS425]

DOM0090 cln3::LEU2

DOM0090 whi5::LEU2

DOM0090 cln3::CLN3- $\triangle C:: H I S 3$

MKy92-1 cln3::CLN3- $\triangle C::$ HIS3

DOM0090 whi5::HIS3

MKy21 WHI5pr-3Flag-WHI5 ${ }^{C D K}$-pRS406

MKy76 cln3::hphMX

DOM0090 CLN3-V5:: hphMX

DOM0090 HIS3pr-GFP-NLS-V5-pRS306::URA3

DOM0090 SWI4-V5:: hphMX

DOM0090 SWI6-V5:: hphMX

MKy554 whi5::URA3

MKy554 stb1::URA3

MKy637 stb1::LEU2

MKy367

MATa ADE2 cln3::TRP1

JE103 CLN3pr-pRS406 


\begin{tabular}{|c|c|c|}
\hline 351 & MKy369 & JE103 CLN3pr -CLN3-pRS406 \\
\hline 352 & MKy372 & JE103 CLN3pr -CLN3 ${ }^{C B M}-p R S 406$ \\
\hline 353 & MKy395 & JE103 CLN3pr -CLN3 ${ }^{C B M}-L-C D K 1-p R S 406$ \\
\hline 354 & MKy386 & JE103 CLN3pr -CLN3 ${ }^{C B M}-L-C D K 1^{K D}-p R S 406$ \\
\hline 55 & MKy320 & DOM0090 cdk1::prGALL-CDK1-kanMX4 \\
\hline 56 & MKy280 & DOM0090 cln3::CLN3-L-CDK1-TRP1 \\
\hline 57 & MKy281 & DOM0090 cln3::CLN3-L-CDK1 $1^{K D}-T R P 1$ \\
\hline 58 & MKy343 & MKy280 clb5::CLB5-L-CDK1-LEU2 \\
\hline 59 & MKy344 & MKy281 clb5::CLB5-L-CDK1-LEU2 \\
\hline 60 & MKy345 & MKy343 clb2::CLB2-L-CDK1-HIS3 \\
\hline 361 & MKy346 & MKy344 clb2::CLB2-L-CDK1-HIS3 \\
\hline 362 & MKy341 & MKy345 cdk1::prGALL-CDK1-kanMX4 \\
\hline 363 & MKy342 & MKy346 cdk1::prGALL-CDK1-kanMX4 \\
\hline 364 & MKy784 & MKy320 whi5::hphMX \\
\hline 65 & MKy785 & MKy341 whi5::hphMX \\
\hline 66 & MKy786 & MKy342 whi5::hphMX \\
\hline 367 & MKy768-1 & DOM0090 kin28::KIN28 ${ }^{i s}-T R P 1$ \\
\hline 368 & MKy783-1 & MKy768-1 cln3::natMX \\
\hline 369 & K14708 & (W303) MATa tor1-1 fpr1::natMX \\
\hline 370 & MK629-1 & K14708 whi5::URA3 \\
\hline 371 & MK630-1 & K14708 cln3::URA3 \\
\hline 32 & MKy631-1 & K14708 SWI6-FKB12::TRP1 \\
\hline 373 & MKy666-1 & MKy631-1 cln3::LEU2 \\
\hline 374 & MKy680-1 & MKy666-1 CLN3pr-CCL1-L-KIN28-Frb-SIVh::HIS3 \\
\hline 375 & MKy681-1 & MKy666-1 CLN3pr-CCL1-L-KIN28-SIVh::HIS3 \\
\hline 376 & MKy803-1 & MKy666-1 CLN3pr-CCL1-L-KIN28 ${ }^{K D}-F r b-S I V h:: H I S 3$ \\
\hline 377 & KSy026-4 & MATa ADE2 bck2::TRP1 cln3::LEU2-MET25pr-CLN3 \\
\hline 378 & MKy708-1 & K14708 SWI4-Fkb12::TRP1 \\
\hline 37 & MKy706-1 & MKy708-1 cln3::LEU2 \\
\hline 380 & MKy719-1 & MKy706-1 CLN3pr-CCL1-L-KIN28-SIVh::HIS3 \\
\hline 381 & MKy720-1 & MKy706-1 CLN3pr-CCL1-L-KIN28-Frb-SIVh::HIS3 \\
\hline
\end{tabular}

Table S2: Plasmids used in this study.

385

386

387

388

389

\section{Plasmid Description}

pMK178

pGAL1-3Flag-CLN3-L-CDK1-pRS425

pMK185

pGAL1-3Flag-CLN3 ${ }^{C B M}$-L-CDK1-pRS425

pMK184

pGAL1-3Flag-CLN2-L-CDK1-pRS425

pMK186

pGAL1-3Flag-CLN1-L-CDK1-pRS425

391

pMK361

pGAL1-3Flag-CLB5-L-CDK1-pRS425

392

pMK362

pGAL1-3Flag-CLB6-L-CDK1-pRS425

pMK362

pGAL1-3Flag-CLB3-L-CDK1-pRS425

394

pMK364

pGAL1-3Flag-CLB4-L-CDK1-pRS425

pMK366

pGAL1-3Flag-CLB2-L-CDK1-pRS425

396

pMK308

pGAL1-3Flag-CCL1-L-KIN28 ${ }^{\text {nointron }}-p R S 425$

397 pMK367

pGAL1-3Flag-BUR2-L-BUR1-pRS425

398

pMK368

pGAL1-3Flag-CTK2-L-CTK1-pRS425

399 pMK369

pGAL1-3Flag-SSN8-L-SSN3-pRS425

$400 \quad$ pMK187

WHI5pr-3Flag-WHI5 ${ }^{C D K}$-pRS406

401 pMK188

CLN3pr-pRS406 


\begin{tabular}{|c|c|c|}
\hline 402 & pMK189 & CLN3pr-CLN3-pRS406 \\
\hline 403 & pMK190 & $C L N 3 p r-C L N 3^{C B M}-p R S 406$ \\
\hline 404 & pMK191 & $C L N 3 p r-C L N 3^{C B M}-L-C D K 1-p R S 406$ \\
\hline 405 & pMK192 & $C L N 3 p r-C L N 3^{C B M}-L-C D K 1^{K D}-p R S 406$ \\
\hline 406 & pMK159 & CLN3 $1214-1740-L-C D K 1-p R S 403$ \\
\hline 407 & pMK160 & $C L N 3_{1214-1740-L-C D K 1^{K D}-p R S 403}$ \\
\hline 408 & pMK161 & CLB5841-1305-L-CDK1-pRS403 \\
\hline 409 & pMK162 & $C L B 2_{721-1473-L-C D K 1-p R S 403}$ \\
\hline 410 & pMK0150 & SIC1dC-pET28a \\
\hline 411 & pMK163 & WHI5-pET28a \\
\hline 412 & pMK300 & BDP1-pET28a \\
\hline 413 & pMK0078 & STB1-pET28a \\
\hline 414 & pMK140 & WHI3-pET28a \\
\hline 415 & pMK141 & SIC1-pET28a \\
\hline 416 & pMK142 & CLN3-pET28 \\
\hline 417 & pMK143 & CLN2-pET28a \\
\hline 418 & pMK144 & RPD3-pET28a \\
\hline 419 & pMK145 & CIP1-pET28a \\
\hline 420 & pMK146 & $C L N 2 d N-p E T 28 a$ \\
\hline 421 & pMK147 & $C L N 3 d N-p E T 28 a$ \\
\hline 422 & pMK370 & $R P B 1 d N-p E T 28 a$ \\
\hline 423 & pMK371 & $R P B 1 d N-R-p E T 28 a$ \\
\hline 424 & pMK372 & $R P B 1 d N-N R-p E T 28 a$ \\
\hline 425 & pMK373 & $R P B 1 d N-R C-p E T 28 a$ \\
\hline 426 & pMK374 & $S P T 5 d N-p E T 28 a$ \\
\hline 427 & pMK148 & WHI7-pET28a \\
\hline 428 & pMK149 & DOA1-pET28a \\
\hline 429 & pMK150 & GCR1-pET28a \\
\hline 430 & pMK151 & BOI2-pET28a \\
\hline 431 & pMK152 & $C D C 48 d N-p E T 28 a$ \\
\hline 432 & pMK153 & YMR147-pET28a \\
\hline 433 & pMK0086 & MSA1-pET28a \\
\hline 434 & pMK165 & GST-WHI5-pGEX-4T-1 \\
\hline 435 & pMK375 & GST-RPB1dN-pGEX-4T-1 \\
\hline 436 & pMK376 & GST-MED15-pGEX-4T-1 \\
\hline 437 & pMK377 & GST-SWI6-pGEX-4T-1 \\
\hline 438 & pMK378 & GST-SWI4-pGEX-4T-1 \\
\hline 439 & pMK379 & GST-RPB1-pGEX-4T-1 \\
\hline 440 & pMK380 & GST-RPB1dC-pGEX-4T-1 \\
\hline 441 & pMK381 & GST-4CTD-pGEX-4T-1 \\
\hline 442 & pMK382 & GST-4CTD-1A-pGEX-4T-1 \\
\hline 443 & pMK383 & GST-4CTD-1F-pGEX-4T-1 \\
\hline 444 & pMK384 & GST-4CTD-2A-pGEX-4T-1 \\
\hline 445 & pMK385 & GST-4CTD-3A-pGEX-4T-1 \\
\hline 446 & pMK386 & GST-4CTD-5A-pGEX-4T-1 \\
\hline 447 & pMK387 & GST-4CTD-6A-pGEX-4T-1 \\
\hline 448 & pMK388 & GST-4CTD-7A-pGEX-4T-1 \\
\hline 449 & pMK389 & GST-4CTD-2/5/7A-pGEX-4T-1 \\
\hline 450 & pMK390 & GST-4CTD-2S-pGEX-4T-1 \\
\hline 451 & pMK358 & CLN3pr-CCL1-L-KIN28-Frb-SIVh \\
\hline 452 & pMK359 & CLN3pr-CCL1-L-KIN28-SIVh \\
\hline
\end{tabular}




\author{
453 pMK360 CLN3pr-CCL1-L-KIN28 ${ }^{K D}-F r b-S I V h$ \\ 454 pMK315 CCL1pr-CCL1-L-KIN28-Frb-SIVh \\ 455 pMK314 CCL1pr-CCL1-L-KIN28-SIVh \\ 456 pMS121 HIS3pr-GFP-NLS-V5-pRS306 \\ 457 pBTC054 GST-H1-pGEX-4T-1 \\ 458 \\ 459 \\ 460 Table S3: List of gene promoters overlapping with Cln3-V5 ChIP-seq peaks and \\ 461 associated peak coordinates. See Methods for details. \\ 462 \\ 463 Table S4: List of substrates and quantification of their phosphorylation by G1 \\ 464 cyclin-Cdk1 complexes.


467

468

469

470

471

472

473

474

475

476

477

478

479

480

481

482

483

484

485

486

487

488

489

490

491

492

493

494

495

496

497

498

499

500

501

502

503

504

505

506

507

508

509

510

511

512

513

514

515

516

517

\section{METHODS}

\section{Yeast strains and plasmids.}

Standard procedures were used for growth and genetic manipulation of Saccharomyces cerevisiae. Cells were grown at $30^{\circ} \mathrm{C}$ in yeast extract/peptone medium with $2 \%$ glucose (YPD) or $2 \%$ galactose (YPGal), or in synthetic complete medium with $2 \%$ glucose (SCD) or $2 \%$ raffinose or with $2 \%$ glycerol and $1 \%$ ethanol. All S. cerevisiae strains in this study are derived from the W303 background. Full genotypes of all strains used in this study are listed in Table S1. Plasmids used in this study are listed in Table S2. For strains to conditionally inactivate Kin28, the endogenous KIN28 gene was replaced with its KIN28 is counterpart by allele replacement into $\operatorname{cln} 3 \Delta$ or wild type backgrounds(36). After recombination, replacement of KIN28 with KIN28 $8^{\text {is }}$ was screened by growing colonies on rich media (YPD) or media containing 5 $\mu \mathrm{M} \mathrm{CMK} \mathrm{(YPD+CMK).} \mathrm{Colonies} \mathrm{that} \mathrm{displayed} \mathrm{a}$ growth defect on YPD+CMK, but not on YPD, were selected and genotyped. For strains to conditionally recruit the Ccl1-L-Kin28 fusion protein to SBF, we used the anchor-away technique(37). A rapamycin-resistant strain background that contained a mutated TOR1 (tor1-1) and deleted FPR1 (fpr1 1 ) was used and the anchor (SWI4 or SWI6) was Cterminally tagged with FKBP12 (human $12 \mathrm{kDa}$ FK506 binding protein) and an extra copy of C-terminally tagged KIN28 was C-terminally tagged with FRB (11 kDa, FKBP12rapamycin-binding domain of human $\mathrm{mTOR}$ ) and expressed ectopically as a fusion with its cyclin partner CCL1 from the CLN3 promoter (CLN3pr-CCL1-L-KIN28-FRB). To induce protein binding, cells were cultured at $30^{\circ} \mathrm{C}$ in SCD media with1 $\mu \mathrm{g} / \mathrm{ml}$ rapamycin, or DMSO as a control.

Cell size measurements. Cell volume was measured using a Beckman Coulter Z2 counter (Beckman Coulter). Log-phase cultures at $\mathrm{OD}_{600}$ 0.2-0.3 were briefly sonicated, and then 100-150 $\mu \mathrm{L}$ was diluted into $10 \mathrm{~mL}$ of Isoton II diluent (Beckman Coulter \#8546719), and 40,000-50,000 cells were measured per sample. Particles below $10 \mathrm{fL}$ and over $300 \mathrm{fL}$ in volume were excluded from analysis.

Immunoblotting. Protein lysates were taken in urea lysis buffer as previously described(31). Protein lysates were separated on tris-glycine or tris-acetate SDS-PAGE gels and transferred to a nitrocellulose membrane using the iBlot 2 dry blotting system (Invitrogen IB21001). The following primary antibodies where used for western-blotting at 1/1000 dilution: anti-Rpb1-CTD clone 8wG16 (mouse, monoclonal, Abcam), anti-Rpb3 clone $1 Y 26$ (mouse, monoclonal, BioLegend), anti-Rpb1-S2-P clone 3E10 (rat, monoclonal, Millipore), anti-Rpb1-S5-P clone H14 (mouse, monoclonal, BioLegend), antiRpb1-S5-P clone 3E8 (rat, monoclonal, Millipore), anti-Rpb1-S7-P clone 3E12 (rat, monoclonal, Millipore), anti-Cdc28 clone yC-20 (goat, polyclonal, Santa Cruz), Pcdc2 (T161) Rabbit antibody (Cell Signaling Technology) or anti-FLAG; clone M2 (mouse, monoclonal, SIGMA). Primary antibodies were detected using the following fluorescently labeled secondary antibodies at 1/10,000 dilution: IRDye 680LT Goat antiMouse, IRDye 680LT Goat anti-Rat, IRDye 800CW Goat anti-Rat, IRDye 800CW Goat anti-Mouse (Licor), Alexa Fluor 680 Donkey anti-Mouse, Alexa Fluor 680 donkey antiRabbit and Alexa Fluor 790 Goat anti-Rabbit (Invitrogen by Thermo Fisher Scientific). Membranes were then imaged on a LI-COR Odyssey CLx.

Immunoblot quantifications. Band intensities where quantified using the LI-COR ImageStudio Lite software. For the quantifications in Fig. 3L, the Rpb1-CTD-S5-P signal was normalized for loading to Rpb3. To calculate the Kin28-dependent fraction of Rpb1CTD-S5-P, the difference between before $(t=0)$ and after CMK addition $(t=20)$ in the WT 
518 (i.e., not $c \ln 3 \Delta$ ) background was calculated and divided through its value at $\mathrm{t}=0$. Then, to 519 calculate the CIn3-dependent Rpb1-CTD-S5-P fraction, the difference between the $\operatorname{cln} 3 \Delta$ and CLN3 wild type background after CMK addition ( $\mathrm{t}=20)$ was calculated and divided through its value at $\mathrm{t}=0$ in the CLN3 wild type background. The mean and S.E.M. were calculated from two independent biological replicates.

Spot viability assay. Plate spot assays show cell growth on YP plate with $2 \%$ glucose or $2 \%$ galactose from a series of culture dilutions $(2 x, 10 x, 5 x$, and $10 x)$ from an initial amount of $10^{\circ}$ cells. Plates were incubated at $30^{\circ} \mathrm{C}$ and photographed at least $40 \mathrm{~h}$ later.

Microscopy. Cells were grown in a CellASIC microfluidic device as previously described(5). For the experiment in Fig. 2E cells were grown in SC medium with $2 \%$ galactose for $2 \mathrm{~h}$ before media was replaced with SC $2 \%$ glucose to turn off the expression of endogenous GAL1pr-CDK1. Phase-contrast images were acquired every 3 minutes after media shift, and multiple fields of view were followed simultaneously. For full movies see supporting material Movies S1-4.

ChIP-seq experiments. Cells expressing CIn3-V5, Swi4-V5, Swi6-V5 or GFP-NLS-V5 were grown in SC media with $2 \%$ glycerol $1 \%$ ethanol. $250 \mathrm{ml}$ of cells at OD $\sim 0.6$ were fixed with $1 \%$ formaldehyde (30 minutes) and quenched with $0.125 \mathrm{M}$ glycine (5 minutes). Fixed cells were washed twice in cold PBS, pelleted, snap-frozen and stored at $-80^{\circ} \mathrm{C}$. Cell lysis and ChIP reactions were performed as previously described(52) with minor modifications. Pellets were lysed in $300 \mu \mathrm{L}$ FA lysis buffer (50 mM HEPES-KOH pH 8.0, $150 \mathrm{mM} \mathrm{NaCl}, 1 \mathrm{mM}$ EDTA, $1 \%$ Triton X-100, 0.1\% sodium deoxycholate, $1 \mathrm{mM}$ PMSF, Roche protease inhibitor) with $\sim 1 \mathrm{~mL}$ ceramic beads on a Fastprep-24 (MP Biomedicals). The entire lysate was then collected and adjusted to $1 \mathrm{~mL}$ before sonication with a 1/8' microtip on a Q500 sonicator (Qsonica) for 16 minutes (10 seconds on, 20 seconds off). The sample tube was held suspended in a $-20^{\circ} \mathrm{C} 80 \%$ ethanol bath to prevent sample heating during sonication. Cell debris was then pelleted and the supernatant retained for ChIP. For each ChIP reaction, $30 \mu \mathrm{L}$ Protein G Dynabeads (Invitrogen) were blocked (PBS $+0.5 \%$ BSA), prebound with 5-10 $\mu \mathrm{L}$ anti-V5 antibody (SV5-Pk1, BioRad Cat\# MCA1360G) and washed once with PBS before incubation with supernatant $\left(4^{\circ} \mathrm{C}\right.$, overnight). Dynabeads were then washed (5 minutes per wash) twice in FA lysis buffer, twice in high-salt FA lysis buffer $(50 \mathrm{mM}$ HepesKOH pH 8.0, $500 \mathrm{mM} \mathrm{NaCl}, 1 \mathrm{mM}$ EDTA, $1 \%$ Triton X-100, $0.1 \%$ sodium deoxycholate, $1 \mathrm{mM}$ PMSF), twice in ChIP wash buffer (10 $\mathrm{mM}$ TrisHCl pH 7.5, $0.25 \mathrm{M} \mathrm{LiCl}, 0.5 \% \mathrm{NP}-40,0.5 \%$ sodium deoxycholate, $1 \mathrm{mM}$ EDTA, $1 \mathrm{mM}$ PMSF) and once in TE wash buffer (10 mM TrisHCl pH 7.5, $1 \mathrm{mM}$ EDTA, $50 \mathrm{mM}$ $\mathrm{NaCl})$. DNA was eluted in ChIP elution buffer $(50 \mathrm{mM}$ TrisHCl pH 7.5, $10 \mathrm{mM}$ EDTA, $1 \%$ SDS) at $65^{\circ} \mathrm{C}$ for 20 minutes. Eluted DNA was incubated to reverse crosslinks $\left(65^{\circ} \mathrm{C}, 5 \mathrm{hr}\right)$, before treatment with RNAse $\mathrm{A}\left(37^{\circ} \mathrm{C}, 1\right.$ hour) and then Proteinase $\mathrm{K}\left(65^{\circ} \mathrm{C}, 2\right.$ hours). DNA was purified using the ChIP DNA Clean \& Concentrator kit (Zymo Research). Indexed sequencing libraries were generated using the NEBNext Ultra II DNA Library Prep kit (NEB Cat \# E7645), pooled and sequenced on an Illumina HiSeq instrument as paired end reads (Novogene, $\mathrm{CA}$ ).

ChIP-seq analysis. Data processing was performed in Galaxy (https://usegalaxy.org/). Reads were trimmed to $36 \mathrm{bp}$ using Cutadapt and then aligned to the $S$. cerevisiae genome (SacCer3) using Bowtie2. RPKM normalized Bigwig files were generated using bam Coverage (bin size $=10 \mathrm{bp}$, paired end reads extended, smoothing $=100 \mathrm{bp}$ ) and used for track display with Integrative Genome Viewer. BAM files were filtered to remove duplicate and low-quality reads with BAM filter before peak calling with MACS2 using 
GFP-NLS-V5 as the control (genome size $=12000000$, bandwidth $=200$ ). Cln3 peaks were defined as regions in which peaks were identified in both CIn3-V5 ChIP replicates $(n=58)$ and SBF peaks were defined as regions in which peaks where identified in Swi4V5 and Swi6-V5 ChIP experiments $(n=311)$. SGD genes where downloaded from UCSC Main in BED format and promoters were defined as $1 \mathrm{~kb}$ upstream of the ORF start. Table S3 contains the list of 85 promoters which overlapped with the $58 \mathrm{Cln} 3$ peaks.

Protein expression and purification. Full-length $\mathrm{N}$-terminally glutathione $\mathrm{S}$-transferasetagged (GST-tagged) proteins were expressed in the E. coli strain BL21 and purified by glutathione-agarose affinity chromatography (Sigma-Aldrich Cat \#G4510) and eluted using elution buffer $(50 \mathrm{mM}$ Tris pH $8.0,100 \mathrm{mM}$ KOAc, $25 \mathrm{mM} \mathrm{MgOAc}, 10 \%$ glycerol, $15 \mathrm{mM}$ glutathione). $\mathrm{N}$-terminally $6 \mathrm{His}$-tagged recombinant substrates were expressed in the E. coli strain BL21 and the purification was performed using cobalt affinity chromatography. Proteins were eluted using buffer containing $50 \mathrm{mM}$ HEPES pH 7.4, $150 \mathrm{mM} \mathrm{NaCl}, 10 \%$ glycerol, and $200 \mathrm{mM}$ imidazole. Histone $\mathrm{H} 1$ protein, which was used as a general substrate for Cdk1, was purchased from EMD Millipore (Cat \#14-155). GST4CTD fusion proteins containing a GST-tag and 4 repeats of the Rpb1 unstructured CTD consensus repeat or repeats with substitutions in specific residues were expressed and purified as described above.

All cyclin-Cdk1 fusion complexes were purified from budding yeast cells using a 3X FLAG affinity purification method modified from a previous protocol used for HA-tag purification(53). Briefly, N-terminally tagged cyclin-Cdk1 fusions were cloned into a pRS425 vector using a glycine-serine linker(24) and overexpressed from the GAL1 budding yeast promoter. The use of a glycine-serine rich linker was a key step in CIn3-LCdk1 purification as the Cln3 protein had notably lower affinity towards Cdk1 in high salt conditions than other S. cerevisiae cyclins. The overexpressed 3X FLAG-tagged cyclinCdk1 complexes were then purified by immunoaffinity chromatography using ANTI-FLAG M2 affinity agarose beads (Sigma-Aldrich Cat \#A2220) and eluted with $0.2 \mathrm{mg} / \mathrm{mL} 3 X$ FLAG peptide (Sigma-Aldrich Cat \#F4799). We note that similar cyclin-Cdk fusions have previously been able to restore wild-type function in vivo(23). Cks1 was purified as described previously(54) and added separately to Cdk1 enzyme complexes in all phosphorylation assays.

In vitro phosphorylation assays. For all phosphorylation assays, equal amounts of substrate and purified kinase complexes were used. Substrate concentrations were kept in the range of $1-5 \mu \mathrm{M}$ for different experiments but did not vary within any experiment. Reaction aliquots were taken at two time points (if not stated otherwise, at the 8-and 16minute time points) and the reaction was stopped with SDS-PAGE sample buffer. The

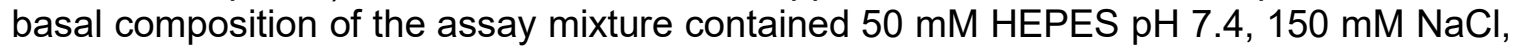
$5 \mathrm{mM} \mathrm{MgCl} 2,0.2 \mathrm{mg} / \mathrm{ml}$ 3X FLAG peptide, $6 \%$ glycerol, $3 \mathrm{mM} \mathrm{EGTA}, 0.2 \mathrm{mg} / \mathrm{ml} \mathrm{BSA}$, and $500 \mu \mathrm{M}$ ATP (with $2 \mu \mathrm{Ci}$ of [Y-32P] ATP added per reaction; PerkinElmer BLU502Z250UC). Phosphorylated proteins were separated on 10\% SDS-PAGE gels unless stated otherwise. In the case of GST-4CTD model substrates, $12 \%$ SDS-PAGE gels were used, while in the case of full length Rpb1 and Rpb1 1 C, $6 \%$ SDS-PAGE gels were used. Phosphorylation of substrate proteins was visualized using autoradiography (Typhoon 9210; GE Healthcare Life Sciences). Autoradiographs were quantified with the ImageQuant TL Software (GE Healthcare Life Sciences).

Baculovirus-based Cyclin-Cdk1-Cks1 complex expression and purification. Baculovirus-based expression of yeast Cyclin-Cdk1-Cks1 complexes was performed 
620 following (4), as described in (55). Briefly, Sf9 insect cells (gift of Tim Stearns) were grown 621 to confluence in a $75 \mathrm{~cm}^{2}$ culture flask in Sf-900 II SFM media (Thermo-Fisher, Waltham,

$622 \mathrm{MA}$ ) and infected with $3 \mathrm{~mL}, 1.5 \mathrm{~mL}$, and $1.5 \mathrm{~mL}$, respectively, of GST-Cdk1, Cln3, and

623 Cks1 baculovirus stocks (Gift of Mike Tyers). For the no-Cln3 control, Cln3 virus was

624 omitted. After 40 hours of infection, cells were harvested, pelleted, and frozen before being

625 used for purification as follows. Frozen insect cell pellets were thawed and resuspended

626 in $2 \mathrm{~mL}$ of ice-cold lysis buffer (50 mM HEPES pH 7.2, $150 \mathrm{mM} \mathrm{NaCl}, 5 \mathrm{mM}$ EDTA, 0.1\%

$627 \mathrm{v} / \mathrm{v}$ NP-40, 10\% v/v glycerol) supplemented with protease inhibitors $(1 \mu \mathrm{g} / \mathrm{mL}$ Pepstatin A,

$6281 \mu \mathrm{g} / \mathrm{mL}$ Leupeptin, >2 KIU/mL Aprotinin, $1 \mathrm{mM}$ Benzamidine, $1 \mu \mathrm{g} / \mathrm{mL}$ Bestatin, $2 \mathrm{mM}$

629 PMSF) and phosphatase inhibitors (50 mM NaF, $1 \mathrm{mM}$ Sodium orthovanadate, $80 \mathrm{mM}$

630 Beta-glycerophosphate). Resuspended cells were incubated for 30 minutes on ice before

631 being centrifuged for 10 minutes at $18,000 \times \mathrm{g}$ at $4^{\circ} \mathrm{C}$. The supernatant was transferred to

632 a new tube and centrifuged for 20 minutes at $28,000 \times \mathrm{g}$ at $4^{\circ} \mathrm{C}$. The cleared lysate was

633 mixed with $200 \mu \mathrm{L}$ of glutathione agarose beads (Sigma-Aldrich, St. Louis, MO) and turned

634 end-over-end for 1.5 hours at $4^{\circ} \mathrm{C}$ before being loaded into a gravity column. The column

635 was washed with 60 volumes of lysis. Before elution, the beads were resuspended in 600

$636 \mu \mathrm{L}$ lysis buffer, and $100 \mu \mathrm{L}$ of the resulting $25 \%$ slurry was removed for use in on-bead

637 kinase reactions. The beads were allowed to re-settle, and protein was eluted from the

638 resulting column twice, using $175 \mu \mathrm{L}$ of lysis buffer $+25 \mathrm{mM}$ glutathione each time. The

639 beads were incubated in elution buffer for 1 hour before the first elution and 1.5 hours

640 before the second elution. Eluates were aliquoted and flash frozen.

642 Kinase assays using glutathione-agarose-bound kinase activity. For each reaction 643 using bead-bound kinase activity, $20 \mu \mathrm{L}$ of the $25 \%$ glutathione-agarose slurry sampled 644 above was centrifuged to give a $5 \mu \mathrm{L}$ bead pellet. The supernatant was removed, and 5 $645 \mu \mathrm{L}$ of $4 \mathrm{x}$ kinase buffer supplemented with radiolabeled ATP, $0.8 \mathrm{mg} / \mathrm{mL}$ BSA, and $100 \mathrm{nM}$ 646 Cks1 was added. The resulting $10 \mu \mathrm{L}$ of $50 \%$ slurry was then mixed with $10 \mu \mathrm{L}$ of substrate solution, as in the kinase assays described above. Tubes were agitated at regular intervals during kinase reactions to keep the beads in suspension, and samples were removed using cut pipet tips, to ensure bead capture.

Acknowledgements: We thank Tim Stearns for Sf9 cells, Mike Tyers for baculovirus stocks, Jennifer Ewald, Kurt Schmoller, David Morgan, Steve Hahn, and Steve Buratowski for yeast strains. We thank Mart Loog, Jim Ferrell, Fred Cross, Peter Pryciak, Aseem Ansari, David Morgan, and Skotheim lab members for constructive feedback. We thank Ben Reyes Topacio for help with enzyme purifications. This work was supported by the NIH (GM092925 and GM115479), the HHMI-Simons (JMS Faculty Scholars Program), the HFSP (postdoctoral fellowship to MK), and the Life Sciences Research Foundation 658 (Simons Foundation Fellowship to MS). 


\section{Figure S1}
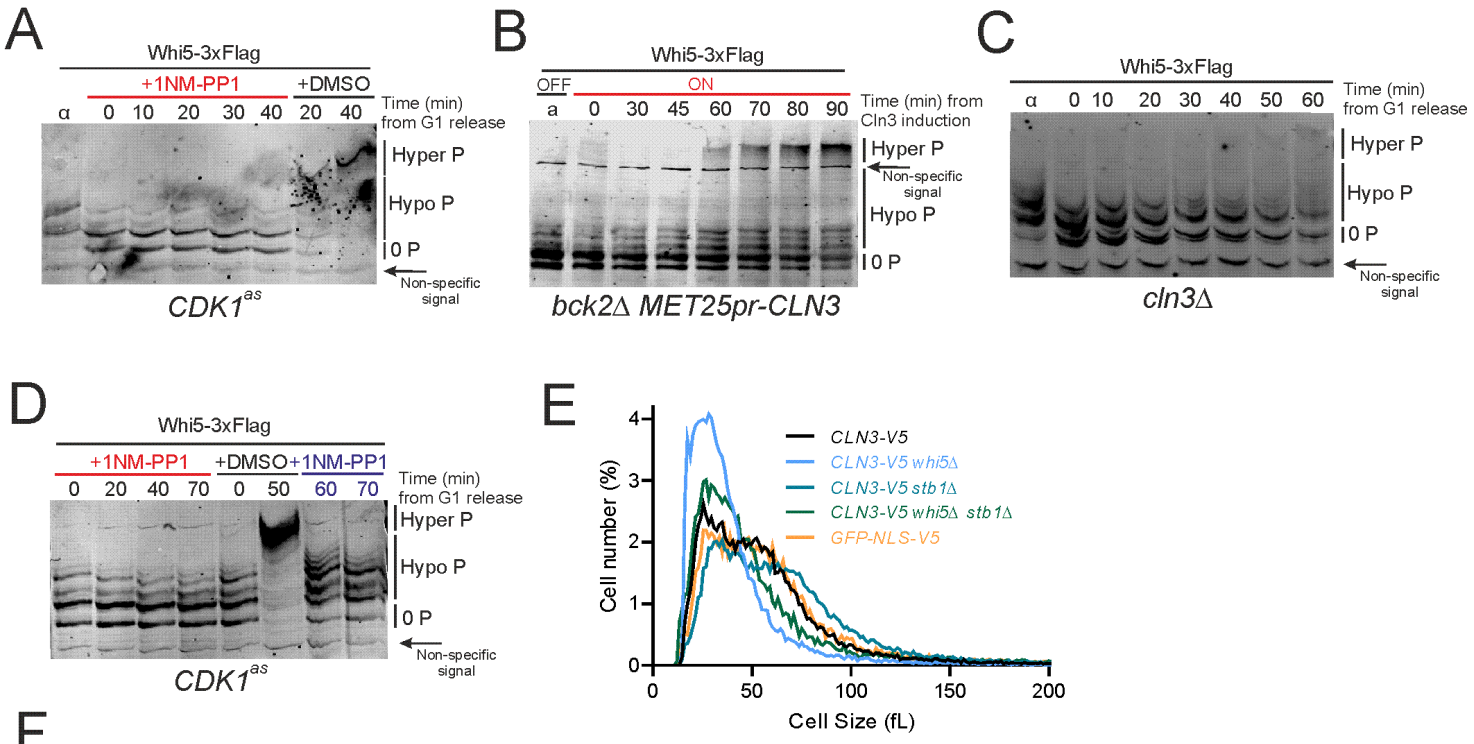

$\mathrm{F}$
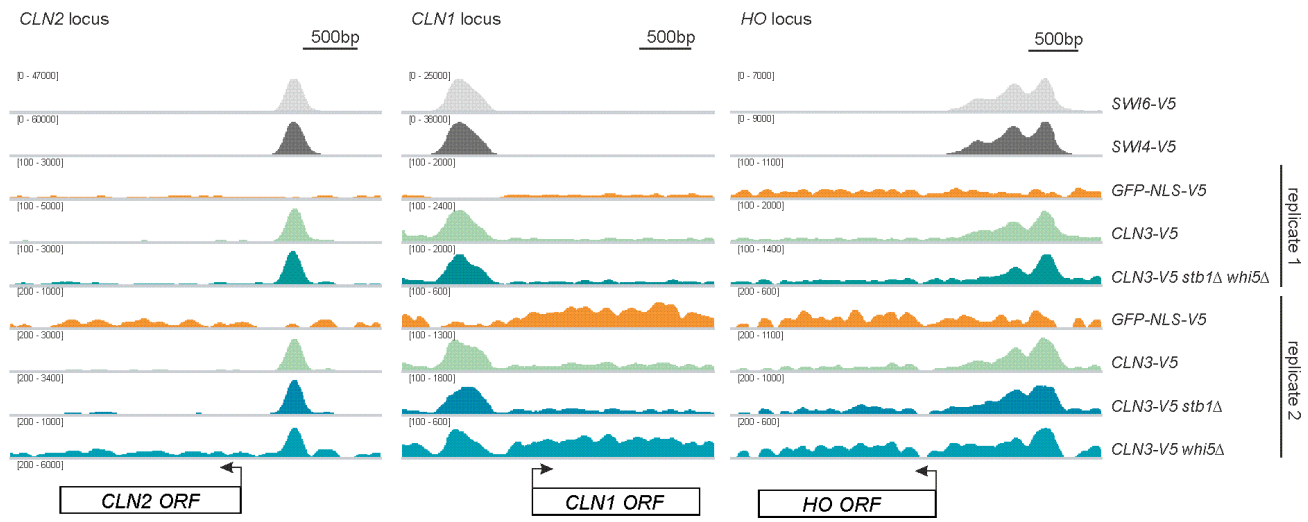

Fig. S1. (A) to (D) Phos-tag immunoblots measuring distinct hypo- and hyperphosphorylated isoforms of Whi5 C-terminally tagged with 3 Flag epitopes expressed from the WHI5 promoter (Whi5-3xFlag). (A) Whi5-3xFlag phosphorylation time series in the ATP analog sensitive CDK1as strain. Cells were released from pheromone-induced G1 arrest into media with DMSO or $10 \mu \mathrm{M}$ of the ATP analog 1NM-PP1. (B) Whi5-3xFlag phosphorylation time series in a bck2 $\Delta$ MET25pr-CLN3 strain. Cells arrested in G1 in the presence of methionine and then were released into media without methionine. (C) Whi5-3xFlag phosphorylation time series after $c \ln 3 \Delta$ cells were released from a pheromone-induced $\mathrm{G} 1$ arrest. (D) Whi5-3xFlag phosphorylation time series in a CDK1 1as strain. Cells were

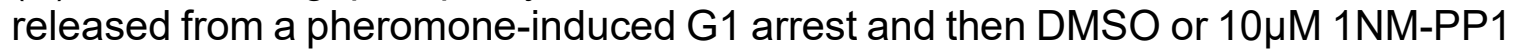
were added at 0 minutes when Whi5 was hypophosphorylated. In the case where DMSO was initially added, we then also added $10 \mu \mathrm{M} 1 \mathrm{NM}-\mathrm{PP} 1$ at 50 minutes when Whi5 is hyperphosphorylated. (E) Cell size distributions measured by Coulter counter for the strains used in the Cln3-V5 ChIP experiments used to generate the data for Fig. 1E and fig. S1F. All strains were grown on synthetic complete media $+2 \%$ glycerol $+1 \%$ ethanol. (F) ChIP-seq signal at three example SBF regulated genes: CLN2, CLN1 and HO. CLN3, SWI4 or SWI6 were tagged at the 
678 endogenous loci with the V5 epitope and anti-V5 ChIP-seq was performed in the 679 indicated genotypes as described in the methods. A subset of these data are also 680 presented in Fig. 1E. 


\section{Figure S2}
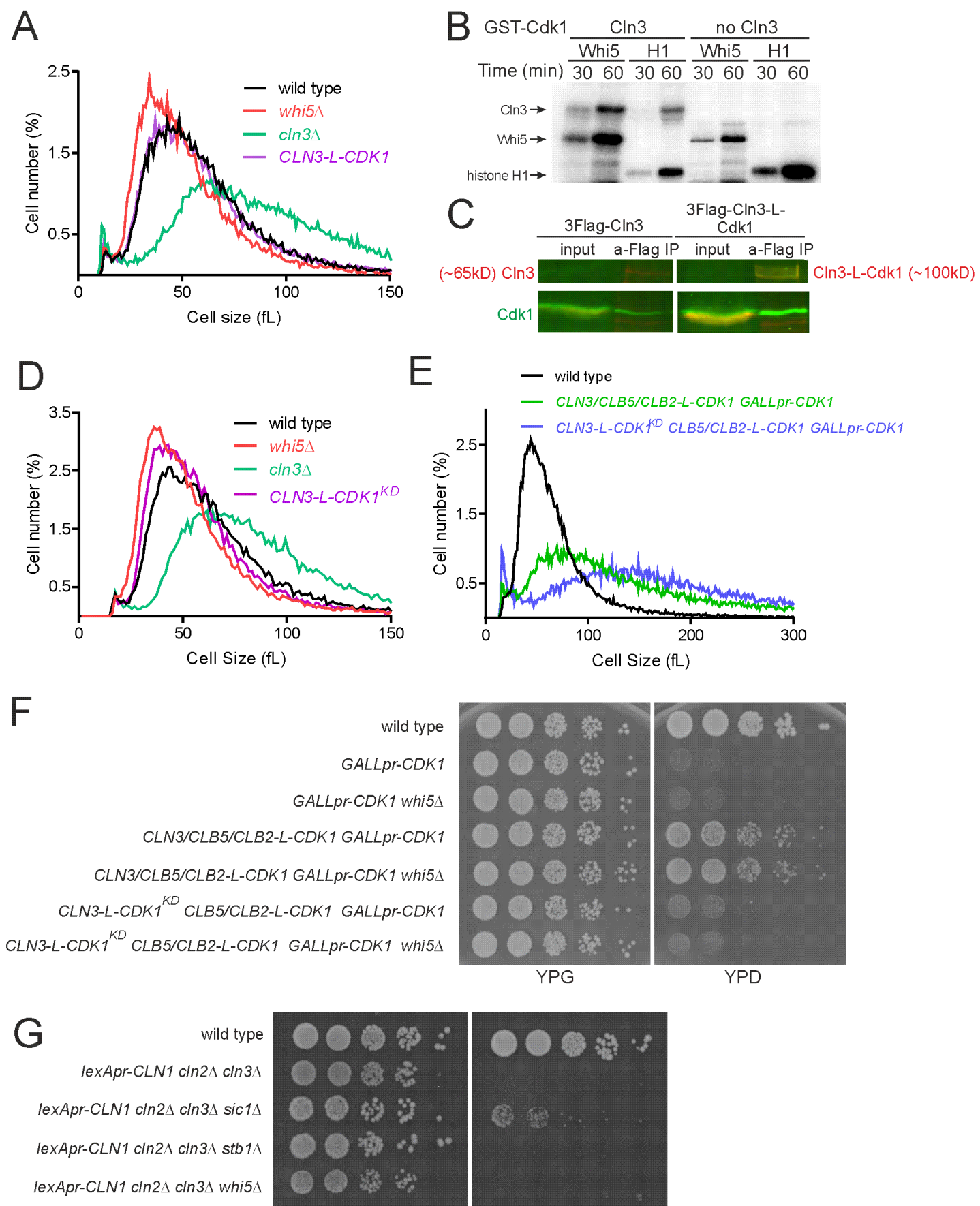

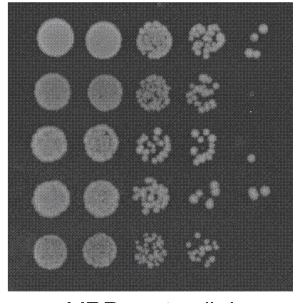

YPD+estradiol

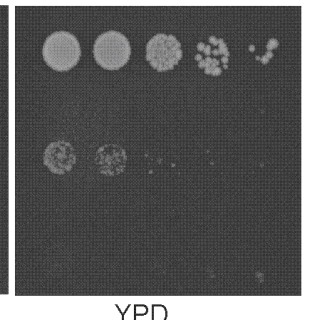

682 Fig. S2. (A) Cell size distributions measured by Coulter counter for the indicated genotypes. Cells were grown on synthetic complete media $+2 \%$ glucose. (B) Autoradiographs of in vitro kinase assays using substrates Whi5 and histone $\mathrm{H} 1$. Kinase activity was purified from Sf9 insect cells expressing Cln3, Cks1 and Cdk1GST (left) or only Cks1 and Cdk1-GST (right). The activity previously reported in (4) to be due to Cln3-Cdk1 in was present in the control purification without Cln3 expression as seen on the right-hand side panel (see methods). (C) Immunoblots 
689 of samples following immunoprecipitation with $3 x F l a g-C \ln 3$ or 3xFlag-CIn3-L$690 \mathrm{Cdk}^{\mathrm{KD}}$, both expressed from the GAL1 promoter. Both 3xFlag-CIn3 and 3xFlag691 Cln3-L-Cdk1 $1^{\mathrm{KD}}$ co-immunoprecipitated with endogenous Cdk1. (D) Cell size 692 distributions, measured by Coulter counter, for the indicated genotypes. Cells were 693 grown on synthetic complete media $+2 \%$ glucose. (E) Cell size distributions, 694 measured by Coulter counter, for the indicated genotypes. Cells were grown on 695 synthetic complete media $+2 \%$ galactose before adding $2 \%$ glucose to repress

696 GALL promoter-dependent expression of Cdk1. Cell size was measured 12 hours 697 after GALLpr repression. (F) Spot viability assays of WT and strains with GALLpr698 dependent expression of Cdk1 on YPG (GALLpr ON) or YPD (GALLpr OFF). 699 Adding three cyclin-Cdk1 fusion genes rescues GALLpr-CDK1 repression. 700 However, if CLN3-CDK1 is replaced with a kinase dead CLN3-CDK1KD fusion, 701 cells are not viable even if WHI5 is deleted. (G) Spot viability assays of WT and 702 strains where $\mathrm{G} 1$ is driven exclusively by the hormone responsive promoter 703 (LexApr) expressing CLN1 on YPD + 50nM Beta-estradiol (LexApr ON) or YPD

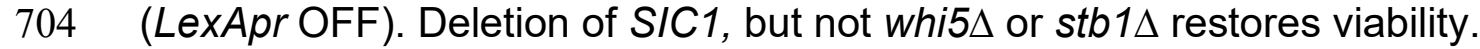




\section{Figure S3}

A

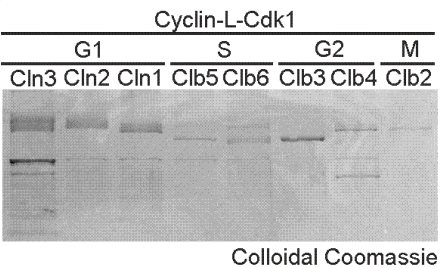

C

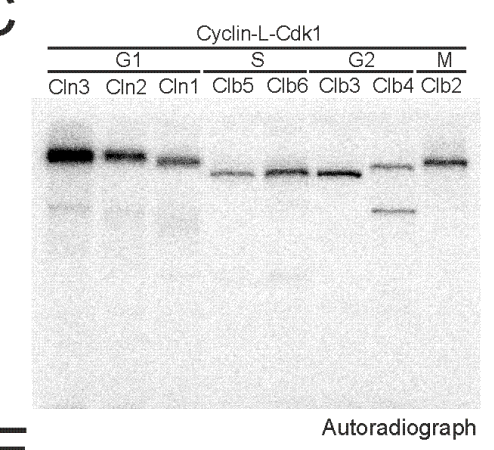

E

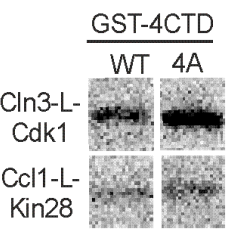

$\mathrm{F}$

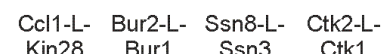

$\mathrm{H}$

anti-Flag

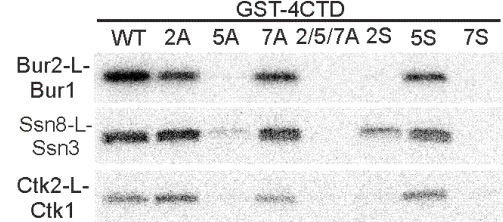

B
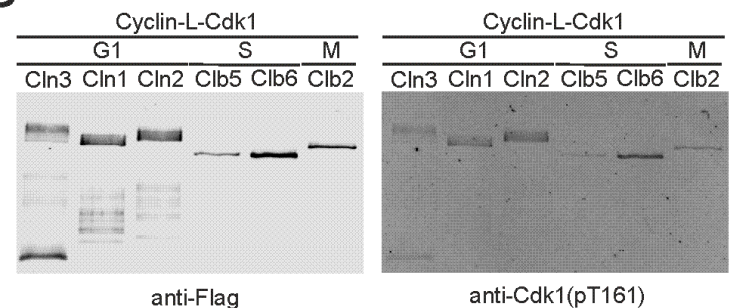

anti-Cdk1(pT161)

D
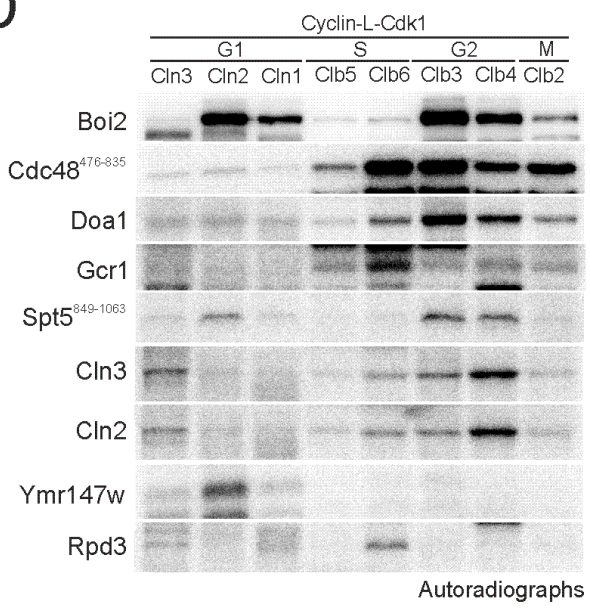

G

$\frac{C \ln 3-L-C d k 1}{\Delta N \quad R \quad R C \quad N R} \frac{C c l 1-L-K i n 28}{\Delta N \quad R \quad R C \quad N R}$

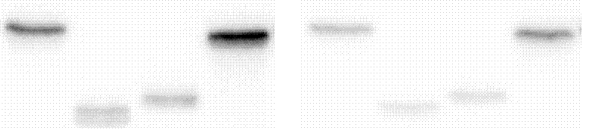

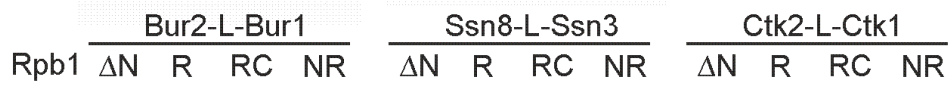

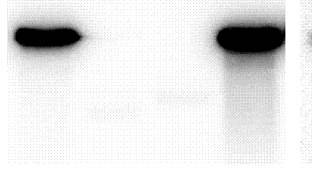

Fig. S3. (A) Colloidal Coomassie-stained SDS gel of all purified cyclin-L-Cdk1 preparations used in this study. (B) Immunoblots to determine the levels (antiFLAG) and activating phosphorylation (anti-Cdk1 pT169) of the indicated purified cyclin-L-Cdk1 complexes used in this study. (C) Autoradiographs of in vitro kinase assays using the indicated purified cyclin-L-Cdk1 complexes without any substrate protein. The signal is due to autophosphorylation. (D) Autoradiographs of in vitro kinase assays using equal amounts of the denoted cyclin-L-Cdk1 and candidate Cln3-Cdk1 substrates. (E) Autoradiographs of in vitro kinase assays using Cln3-LCdk1 and Ccl1-L-Kin28 to phosphorylate a synthetic substrate containing 4 CTD repeats or 4 CTD repeats with threonine 4 mutated to alanine. $(F)$ Immunoblots to 
716 determine the levels (anti-FLAG) of purified transcriptional cyclin-L-Cdk complexes 717 used in this study. (G) Autoradiographs of in vitro kinase assays with transcriptional 718 cyclin-L-Cdk complexes phosphorylating $\mathrm{Rpb} 1 \Delta \mathrm{N}$ and a series of $\mathrm{Rpb} 1 \Delta \mathrm{N}$ 719 truncations. See Fig. 3E for details of truncations. (H) Autoradiographs of in vitro 720 kinase assays using Cln3-L-Cdk1 or transcriptional cyclin-L-Cdks to phosphorylate 721 WT or mutant versions of 4 CTD repeats. See Fig. 3F for details of CTD repeat 722 variants. 
Figure S4
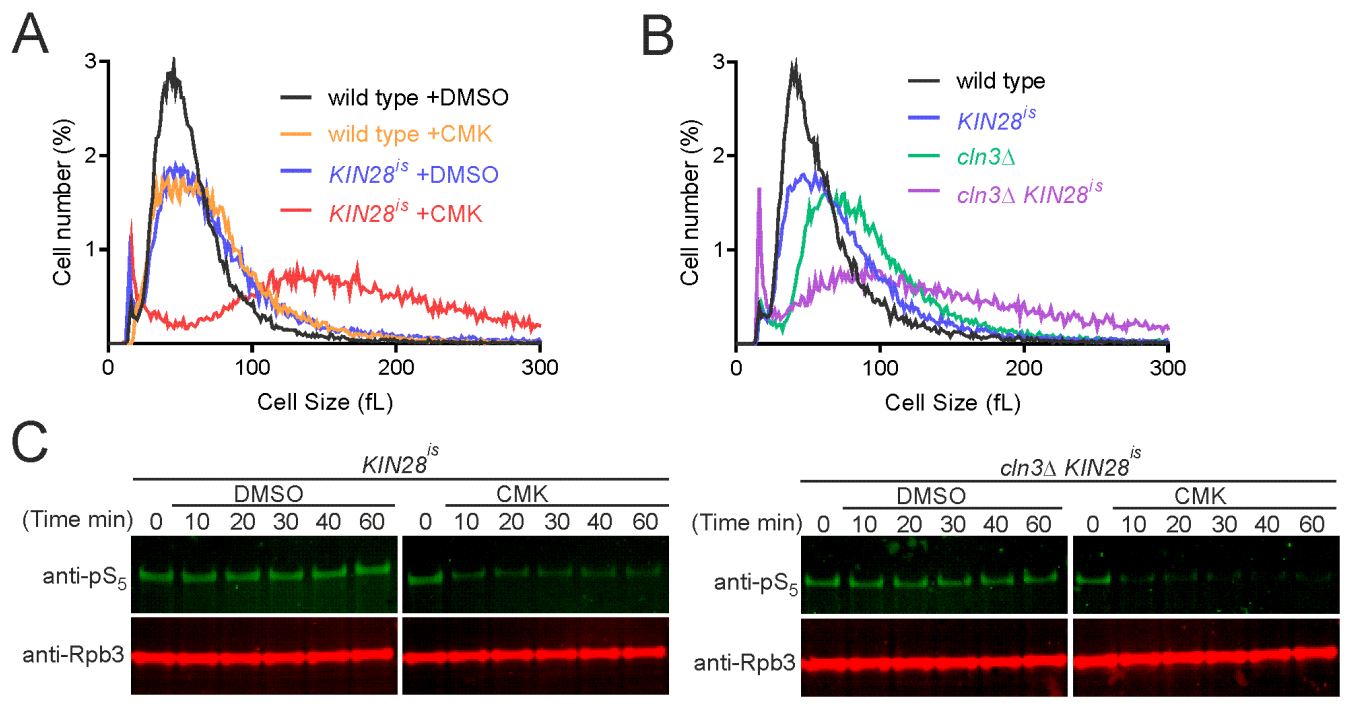

D

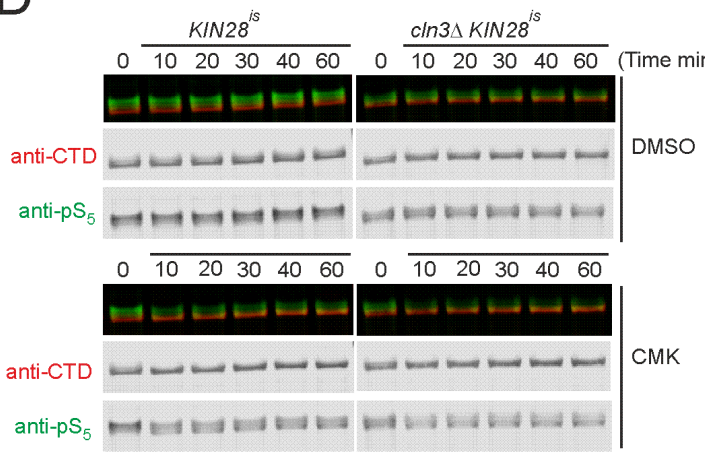

F

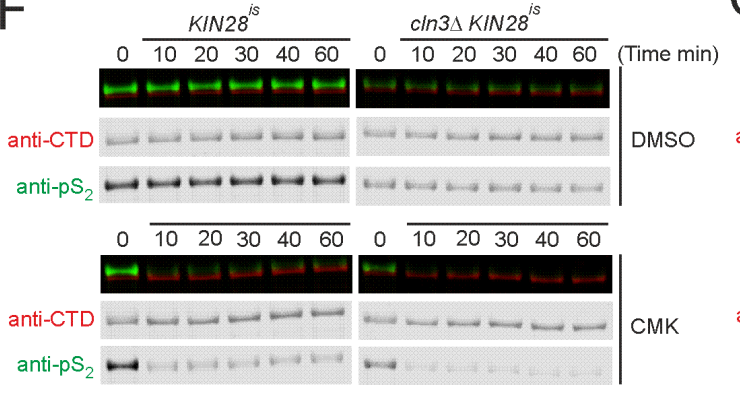

$E$

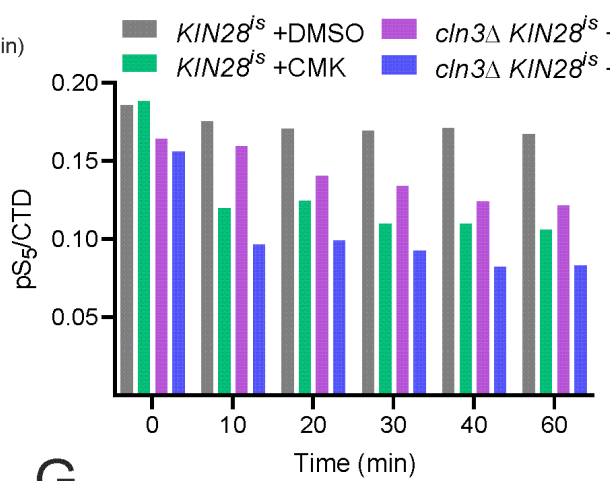

G

KIN $28^{i s} \quad \ln 31 K I N 28^{i s}$

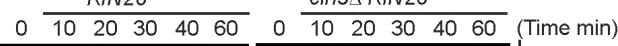

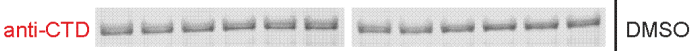
anti-pS

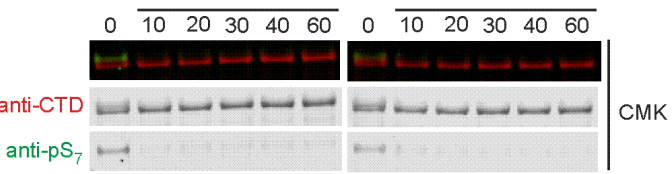

Fig. S4. (A) Cell size distributions measured by Coulter counter for the indicated genotypes. All strains were grown on synthetic complete media $+2 \%$ glucose with $5 \mu \mathrm{M}$ CMK or DMSO. (B) Cell size distributions measured by Coulter counter for the indicated genotypes. All strains were grown on synthetic complete media $+2 \%$ glucose. (C) to (G) Immunoblots and quantifications of different phosphorylated forms of Rpb1 in KIN28is or KIN28 is $\operatorname{cln} 3 \Delta$ strains at the indicated timepoints after release from $\mathrm{G} 1$ pheromone arrest into DMSO or $5 \mu \mathrm{M} \mathrm{CMK}$. Kin28 is contains an active site mutant rendering it sensitive to covalent inhibition by the small molecule CMK. (C) Immunoblots of total cellular phosphorylated Rpb1-CTD $\mathrm{S}_{5}(\mathrm{H} 14$ antibody) and total cellular RNA polymerase II (Rpb3) after release from G1 
735 pheromone arrest into DMSO or $5 \mu \mathrm{M}$ CMK. A subset of these data are also 736 presented in Fig. 3K. (D) Immunoblots of total cellular phosphorylated Rpb1-CTD 737 S5 (3E8 antibody) and Rpb1-CTD (8wG16 antibody) after release from G1 738 pheromone arrest into DMSO or $5 \mu \mathrm{M} \mathrm{CMK}$. (E) Quantification of immunoblots in 739 (D): total cellular phosphorylated CTD $S_{5}$ (3E8 antibody) normalized to Rpb1-CTD 740 (8wG16 antibody). (F) Immunoblots of total cellular phosphorylated Rpb1-CTD S 2 741 (3E10 antibody) and Rpb1-CTD (8wG16 antibody) after release from G1 742 pheromone arrest into DMSO or $5 \mu \mathrm{M}$ CMK. (G) Immunoblots of total cellular 743 phosphorylated Rpb1-CTD $\mathrm{S}_{7}$ (3E12 antibody) and Rpb1-CTD (8wG16 antibody) 744 after release from $\mathrm{G} 1$ pheromone arrest into DMSO or $5 \mu \mathrm{M}$ CMK. 


\section{Figure S5}
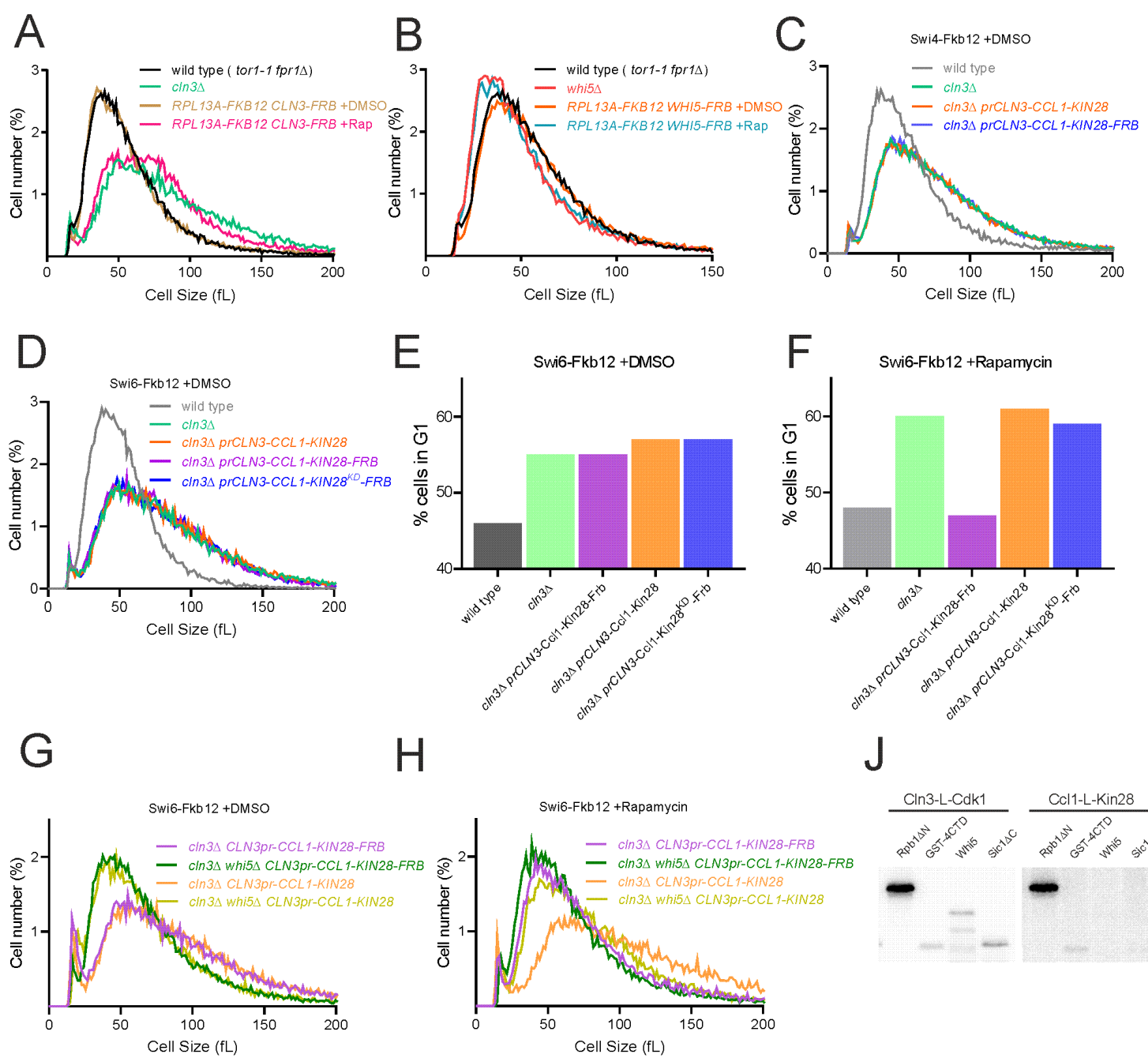

$\mathrm{H}$
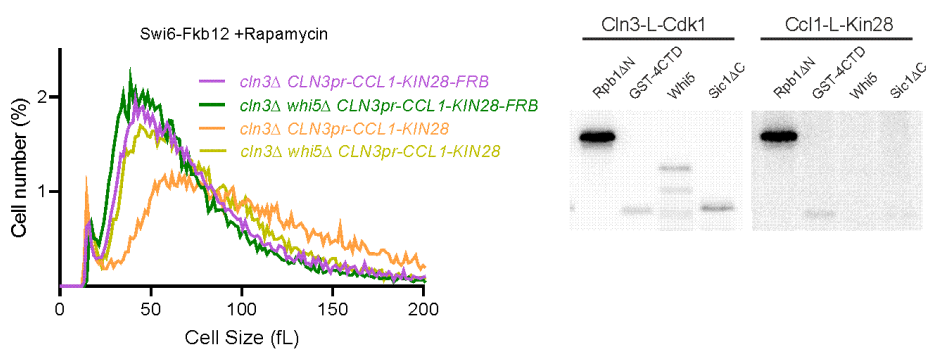

745

Fig. S5. (A) to (B) Cell size distributions measured by Coulter counter for the indicated genotypes. All strains were grown on synthetic complete media $+2 \%$ glucose. $1 \mu \mathrm{g} / \mathrm{ml}$ rapamycin or DMSO was added $\sim 200$ minutes before cell size measurements. (C) to $(\mathbf{H})$ Conditional recruitment of Ccl1-L-Kin28 to SBF using the rapamycin inducible binding system. Ccl1-L-Kin28 fusion proteins were expressed from a genomically integrated copy of the CLN3 promoter. Ccl1-LKin28-FRB was recruited to SBF via its Swi4 (C\&G) or Swi6 (D to $F \& H$ ) subunits upon rapamycin treatment. Ccl1-L-Kin28 lacking FRB is not recruited. All strains were grown on synthetic complete media $+2 \%$ glucose with $1 \mu \mathrm{g} / \mathrm{ml}$ rapamycin or DMSO. (C) to (D) Cell size distributions measured by Coulter counter for the indicated genotypes. (E) to (F) Quantification of flow cytometry analysis of DNA content of the cells in (D) and Fig. 5D. Samples were collected from the same cultures at the same time for both cell size and DNA content measurements. (G) to $(\mathbf{H})$ Cell size distributions measured using a Coulter counter for the indicated genotypes. (J) Autoradiographs of in vitro phosphorylation of Rpb1 $\Delta \mathrm{N}$, GST- 
bioRxiv preprint doi: https://doi.org/10.1101/2021.03.25.436872; this version posted March 25, 2021. The copyright holder for this preprint

(which was not certified by peer review) is the author/funder, who has granted bioRxiv a license to display the preprint in perpetuity. It is made available under aCC-BY-NC-ND 4.0 International license.

761 4CTD, Whi5 or Sic1 $\Delta$ C by Cln3-L-Cdk1 or Ccl1-L-Kin28. Ccl1-L-Kin28 is not able 762 to phosphorylate Whi5. 
763

764

765

766

767

768

769

770

771

772

773

774

775

776

777

778

779

780

781

782

783

784

785

786

787

788

789

790

791

792

793

794

795

796

797

798

799

\section{REFERENCES}

1. C. Bertoli, J. M. Skotheim, R. A. M. de Bruin, Control of cell cycle transcription during G1 and S phases. Nature Reviews Molecular Cell Biology. 14, 518-528 (2013).

2. D. O. Morgan, The Cell Cycle (London, 2007).

3. R. A. M. de Bruin, W. H. McDonald, T. I. Kalashnikova, J. Yates, C. Wittenberg, Cln3 activates G1-specific transcription via phosphorylation of the SBF bound repressor Whi5. Cell. 117, 887-898 (2004).

4. M. Costanzo et al., CDK activity antagonizes Whi5, an inhibitor of G1/S transcription in yeast. Cell. 117, 899-913 (2004).

5. A. Doncic, M. Falleur-Fettig, J. M. Skotheim, Distinct Interactions Select and Maintain a Specific Cell Fate. Mol Cell. 43, 528-539 (2011).

6. J. M. Skotheim, S. Di Talia, E. D. Siggia, F. R. Cross, Positive feedback of G1 cyclins ensures coherent cell cycle entry. Nature. 454, 291-296 (2008).

7. M. Tyers, G. Tokiwa, B. Futcher, Comparison of the Saccharomyces cerevisiae G1 cyclins: $C \ln 3$ may be an upstream activator of $C \ln 1, C \ln 2$ and other cyclins. The EMBO Journal. 12, 1955-1968 (1993).

8. H. Wang, L. B. Carey, Y. Cai, H. Wijnen, B. Futcher, Recruitment of Cln3 Cyclin to Promoters Controls Cell Cycle Entry via Histone Deacetylase and Other Targets. PLoS Biol. 7, e1000189 (2009).

9. J. L. Corden, RNA polymerase II C-terminal domain: Tethering transcription to transcript and template. Chemical Reviews. 113, 8423-8455 (2013).

10. C. Schwarz et al., A Precise Cdk Activity Threshold Determines Passage through the Restriction Point. Mol Cell. 69, 253-264.e5 (2018).

11. S. L. Spencer et al., The proliferation-quiescence decision is controlled by a bifurcation in CDK2 activity at mitotic exit. Cell. 155, 369-383 (2013).

12. S. D. Cappell, M. Chung, A. Jaimovich, S. L. Spencer, T. Meyer, Irreversible APC(Cdh1) Inactivation Underlies the Point of No Return for Cell-Cycle Entry. Cell. 166, 167-180 (2016).

13. A. B. Pardee, $\mathrm{G} 1$ events and regulation of cell proliferation. Science. 246, 603608 (1989).

14. G. Yao, T. J. Lee, S. Mori, J. R. Nevins, L. You, A bistable Rb-E2F switch underlies the restriction point. Nat Cell Biol. 10, 476-482 (2008).

15. C. J. Sherr, The Pezcoller lecture: cancer cell cycles revisited. Cancer Res. 60 , 3689-3695 (2000). 
800 16. A. M. Narasimha et al., Cyclin D activates the Rb tumor suppressor by monophosphorylation. Elife. 3, e02872 (2014).

802 17. B. R. Topacio et al., Cyclin D-Cdk4,6 Drives Cell-Cycle Progression via the Retinoblastoma Protein's C-Terminal Helix. Mol Cell. 74, 758-770.e4 (2019).

804

18. E. Kinoshita, E. Kinoshita-Kikuta, K. Takiyama, T. Koike, Phosphate-binding tag, a new tool to visualize phosphorylated proteins. Mol. Cell Proteomics. 5, 749-757 (2006).

19. S. Bhaduri et al., A docking interface in the cyclin Cln2 promotes multi-site phosphorylation of substrates and timely cell-cycle entry. Curr Biol. 25, 316-325 (2015).

20. K. M. Schmoller, J. J. Turner, M. Kõivomägi, J. M. Skotheim, Dilution of the cell cycle inhibitor Whi5 controls budding-yeast cell size. Nature. 526, 268-272 (2015).

21. R. Nash, G. Tokiwa, S. Anand, K. Erickson, A. B. Futcher, The WHI1+ gene of Saccharomyces cerevisiae tethers cell division to cell size and is a cyclin homolog. The EMBO Journal. 7, 4335-4346 (1988).

22. F. R. Cross, DAF1, a mutant gene affecting size control, pheromone arrest, and cell cycle kinetics of Saccharomyces cerevisiae. Molecular and cellular biology. $\mathbf{8}$, 4675-4684 (1988).

23. D. Coudreuse, P. Nurse, Driving the cell cycle with a minimal CDK control network. Nature. 468, 1074-1079 (2010).

24. R. N. Rao et al., Conditional transformation of rat embryo fibroblast cells by a cyclin D1-cdk4 fusion gene. Oncogene. 18, 6343-6356 (1999).

25. R. A. M. de Bruin, T. I. Kalashnikova, C. Wittenberg, Stb1 collaborates with other regulators to modulate the G1-specific transcriptional circuit. Molecular and cellular biology. 28, 6919-6928 (2008).

26. M. Ashe et al., The SBF- and MBF-associated protein Msa1 is required for proper timing of G1-specific transcription in Saccharomyces cerevisiae. J Biol Chem. 283, 6040-6049 (2008).

27. S. Miles, M. W. Croxford, A. P. Abeysinghe, L. L. Breeden, Msa1 and Msa2 Modulate G1-Specific Transcription to Promote G1 Arrest and the Transition to Quiescence in Budding Yeast. PLoS Genet. 12, e1006088 (2016).

28. H. Wijnen, A. Landman, B. Futcher, The $\mathrm{G}(1)$ cyclin Cln3 promotes cell cycle entry via the transcription factor Swi6. Molecular and cellular biology. 22, 44024418 (2002).

29. L. Kao et al., Global analysis of cdc14 dephosphorylation sites reveals essential regulatory role in mitosis and cytokinesis. - PubMed - NCBI. Mol. Cell Proteomics. 13, 594-605 (2014). 
838 30. M. E. Miller, F. R. Cross, A. L. Groeger, K. L. Jameson, Identification of novel and

839

840

841

842

844 conserved functional and structural elements of the $\mathrm{G} 1$ cyclin Cln3 important for interactions with the CDK Cdc28 in Saccharomyces cerevisiae. Yeast. 22, 10211036 (2005).

31. M. Kõivomägi et al., Dynamics of Cdk1 Substrate Specificity during the Cell Cycle. Mol Cell. 42, 610-623 (2011).

32. K. M. Harlen, L. S. Churchman, The code and beyond: transcription regulation by the RNA polymerase II carboxy-terminal domain. Nature Reviews Molecular Cell Biology. 18, 263-273 (2017).

33. J.-P. Hsin, J. L. Manley, The RNA polymerase II CTD coordinates transcription and RNA processing. Genes \& development. 26, 2119-2137 (2012).

34. Y. Chun et al., Selective kinase inhibition shows that Bur1 (Cdk9) phosphorylates the Rpb1 linker in vivo. Molecular and cellular biology. 39, 395 (2019).

35. P. Chymkowitch et al., Cdc28 kinase activity regulates the basal transcription machinery at a subset of genes. Proc Natl Acad Sci USA. 109, 10450-10455 (2012).

36. J. B. Rodríguez-Molina, S. C. Tseng, S. P. Simonett, J. Taunton, A. Z. Ansari, Engineered Covalent Inactivation of TFIIH-Kinase Reveals an Elongation Checkpoint and Results in Widespread mRNA Stabilization. Mol Cell. 63, 433444 (2016).

37. H. Haruki, J. Nishikawa, U. K. Laemmli, The anchor-away technique: rapid, conditional establishment of yeast mutant phenotypes. Mol Cell. 31, 925-932 (2008).

38. Y. Qu et al., Cell Cycle Inhibitor Whi5 Records Environmental Information to Coordinate Growth and Division in Yeast. Cell Rep. 29, 987-994.e5 (2019).

39. G. Tokiwa, M. Tyers, T. Volpe, B. Futcher, Inhibition of $\mathrm{G} 1$ cyclin activity by the Ras/cAMP pathway in yeast. Nature. 371, 342-345 (1994).

40. M. D. Baroni, P. Monti, L. Alberghina, Repression of growth-regulated G1 cyclin expression by cyclic AMP in budding yeast. Nature. 371, 339-342 (1994).

41. M. Polymenis, E. V. Schmidt, Coupling of cell division to cell growth by translational control of the G1 cyclin CLN3 in yeast. Genes \& development. 11, 2522-2531 (1997).

42. X. Liu et al., Reliable cell cycle commitment in budding yeast is ensured by signal integration. Elife. 4, e03977 (2015).

43. R. Lucena et al., Cell Size and Growth Rate Are Modulated by TORC2-Dependent Signals. Curr Biol. 28, 196-210.e4 (2018). 
874 44. E. M. Medina, J. J. Turner, R. Gordân, J. M. Skotheim, N. E. Buchler, Punctuated

875

876

877 evolution and transitional hybrid network in an ancestral cell cycle of fungi. Elife. 5, 120 (2016).

45. L. Cao et al., Phylogenetic analysis of CDK and cyclin proteins in premetazoan lineages. BMC Evol Biol. 14, 1-16 (2014).

46. D. Hermand et al., Specificity of Cdk activation in vivo by the two Caks Mcs6 and Csk1 in fission yeast. The EMBO Journal. 20, 82-90 (2001).

47. S. Larochelle, J. Pandur, R. P. Fisher, H. K. Salz, B. Suter, Cdk7 is essential for mitosis and for in vivo Cdk-activating kinase activity. Genes \& development. 12, 370-381 (1998).

48. S. Larochelle et al., Requirements for Cdk7 in the assembly of Cdk1/cyclin B and activation of Cdk2 revealed by chemical genetics in human cells. Mol Cell. 25, 839-850 (2007).

49. L. J. Cisek, J. L. Corden, Phosphorylation of RNA polymerase by the murine homologue of the cell-cycle control protein cdc2. Nature. 339, 679-684 (1989).

50. J. C. Ewald, A. kuehne, N. Zamboni, J. M. Skotheim, The Yeast Cyclin-Dependent Kinase Routes Carbon Fluxes to Fuel Cell Cycle Progression. Mol Cell. 62, 532545 (2016).

51. M. Kõivomägi et al., Cascades of multisite phosphorylation control Sic1 destruction at the onset of S phase. Nature. 480, 128-131 (2011).

52. B. Hu et al., Biological chromodynamics: a general method for measuring protein occupancy across the genome by calibrating ChIP-seq. Nucleic Acids Res. 43, e132 (2015).

53. D. McCusker et al., Cdk1 coordinates cell-surface growth with the cell cycle. Nat Cell Biol. 9, 506-515 (2007).

54. G. J. Reynard, W. Reynolds, R. Verma, R. J. Deshaies, Cks1 is required for G(1) cyclin-cyclin-dependent kinase activity in budding yeast. Molecular and cellular biology. 20, 5858-5864 (2000).

55. D. Skowyra, K. L. Craig, M. Tyers, S. J. Elledge, J. W. Harper, F-Box Proteins Are Receptors that Recruit Phosphorylated Substrates to the SCF Ubiquitin-Ligase Complex. Cell. 91, 209-219 (1997). 